\title{
Giant virus diversity and host interactions through global metagenomics
}

https://doi.org/10.1038/s41586-020-1957-x

Received: 4 June 2019

Accepted: 9 January 2020

Published online: 22 January 2020

\section{Open access}

Check for updates

\author{
Frederik Schulz ${ }^{1 凶}$, Simon Roux ${ }^{1}$, David Paez-Espino', Sean Jungbluth', David A. Walsh², \\ Vincent J. Denef ${ }^{3}$, Katherine D. McMahon ${ }^{4,5}$, Konstantinos T. Konstantinidis ${ }^{6}$, \\ Emiley A. Eloe-Fadrosh', Nikos C. Kyrpides ${ }^{1} \&$ Tanja Woyke ${ }^{1 凶}$
}

\begin{abstract}
Our current knowledge about nucleocytoplasmic large DNA viruses (NCLDVs) is largely derived from viral isolates that are co-cultivated with protists and algae. Here we reconstructed 2,074 NCLDV genomes from sampling sites across the globe by building on the rapidly increasing amount of publicly available metagenome data. This led to an 11-fold increase in phylogenetic diversity and a parallel 10 -fold expansion in functional diversity. Analysis of 58,023 major capsid proteins from large and giant viruses using metagenomic data revealed the global distribution patterns and cosmopolitan nature of these viruses. The discovered viral genomes encoded a wide range of proteins with putative roles in photosynthesis and diverse substrate transport processes, indicating that host reprogramming is probably a common strategy in the NCLDVs. Furthermore, inferences of horizontal gene transfer connected viral lineages to diverse eukaryotic hosts. We anticipate that the global diversity of NCLDVs that we describe here will establish giant viruses-which are associated with most major eukaryotic lineages-as important players in ecosystems across Earth's biomes.
\end{abstract}

Large and giant viruses of the NCLDV supergroup have complex genomes with sizes of up to several megabases, and virions that are a similar size to, or even larger than, small cellular organisms $\mathrm{s}^{1-3}$. These viruses infect a wide range of eukaryotes from protists to animals ${ }^{4}$. Marker gene surveys have shown that NCLDVs are not only extremely abundant and diverse in ocean $\mathrm{s}^{5-7}$, but can also frequently be found in freshwater ${ }^{8}$ and soil ${ }^{9}$. However, the discovery of large and giant viruses has mainly been driven by their co-cultivation with amoebae or isolation together with their native hosts ${ }^{1,4,8}$. Only recently, metagenomic and single-cell genomic studies have facilitated the discovery of several new NCLDV members and showed that cultivation-independent methods are applicable to these viruses just as they are to uncultivated Bacteria and Archaea ${ }^{9-14}$.

Here, we have used a multistep metagenome data-mining, binning and iterative-filtering pipeline (Extended Data Figs. 1, 2 and Supplementary Text 1 ), which led to the recovery of genomes representing 2,074 putative NCLDV populations from 8,535 publicly available metagenomes in the Integrated Microbial Genomes and Microbiomes (IMG/M) database ${ }^{15}$. The assembly size, GC content, coding density and copy number of nucleocytoplasmic virus orthologous genes (NCVOGs) ${ }^{16}$ were comparable to previously described NCLDV genomes, supporting the classification of these genomes as giant virus metagenome-assembled genomes (GVMAGs) (Extended Data Figs. 3, 4 and Supplementary Tables 1-3). Using an approach that relied on conserved NCVOGs, we estimated genome completeness and contamination, which led to the classification of 773 high-quality, 989 medium-quality and 312 low-quality GVMAGs (Extended
Data Figs. 1, 4 and Supplementary Tables 1, 4), in line with the MIUViG recommendations ${ }^{17}$.

Augmenting the existing NCLDV phylogenetic framework with the GVMAGs substantially increased the diversity of this proposed viral order (Fig. 1a and Supplementary Data 1). The resulting phylogenetic tree expanded from 205 to 2,279 viral genomes, which can now be divided into 100 potentially genus- or subfamily-level monophyletic clades spanning 10 provisional superclades, compared with the previously recognized 20 genera $^{2}$. This translates into an 11 -fold increase in phylogenetic diversity of the NCLDVs. Notably, the addition of the novel viral genomes did not change the basic topology of the NCLDV tree but rather altered the contribution of existing groups, the Mimiviridae in particular, to the total viral diversity. Furthermore, the presence of conserved NCVOGs in lineage-specific patterns strengthens the hypothesis of a common evolutionary origin of this viral group ${ }^{2}$. Novel groups of viruses with no isolate representatives appeared within the existing taxonomic framework (that is, metagenomic giant virus lineages (MGVLs)). The greatest number of GVMAGs could be attributed to MGVL57 ( $n=205)$, the Yellowstone Lake mimiviruses (YLMVs; $n=119$ ) and MGVL42 $(n=84)$. In addition, several established viral lineages were considerably extended, such as the prasinoviruses $(n=77)$, iridoviruses $(n=59)$, cafeteriaviruses $(n=43)$, phaeocystisviruses $(n=37)$, klosneuviruses $(n=36)$, tetraselmisviruses $(n=34)$ and raphidoviruses $(n=26)$, some of which previously consisted of single isolates. In total, the GVMAGs increased the 123,000 previously known NCLDV proteins that clustered in 47,700 protein families to more than 924,000 proteins in 508,000 protein families (Extended Data Fig. 5a). Pfam-A protein

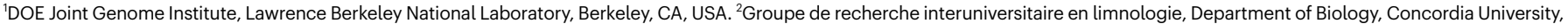
Montréal, Québec, Canada. ${ }^{3}$ Department of Ecology and Evolutionary Biology, University of Michigan, Ann Arbor, MI, USA. ${ }^{4}$ Department of Bacteriology, University of Wisconsin-Madison,

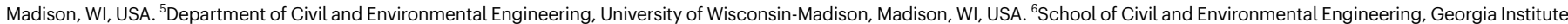
of Technology, Atlanta, GA, USA. ${ }^{凶}$ e-mail: fschulz@lbl.gov; twoyke@lbl.gov 


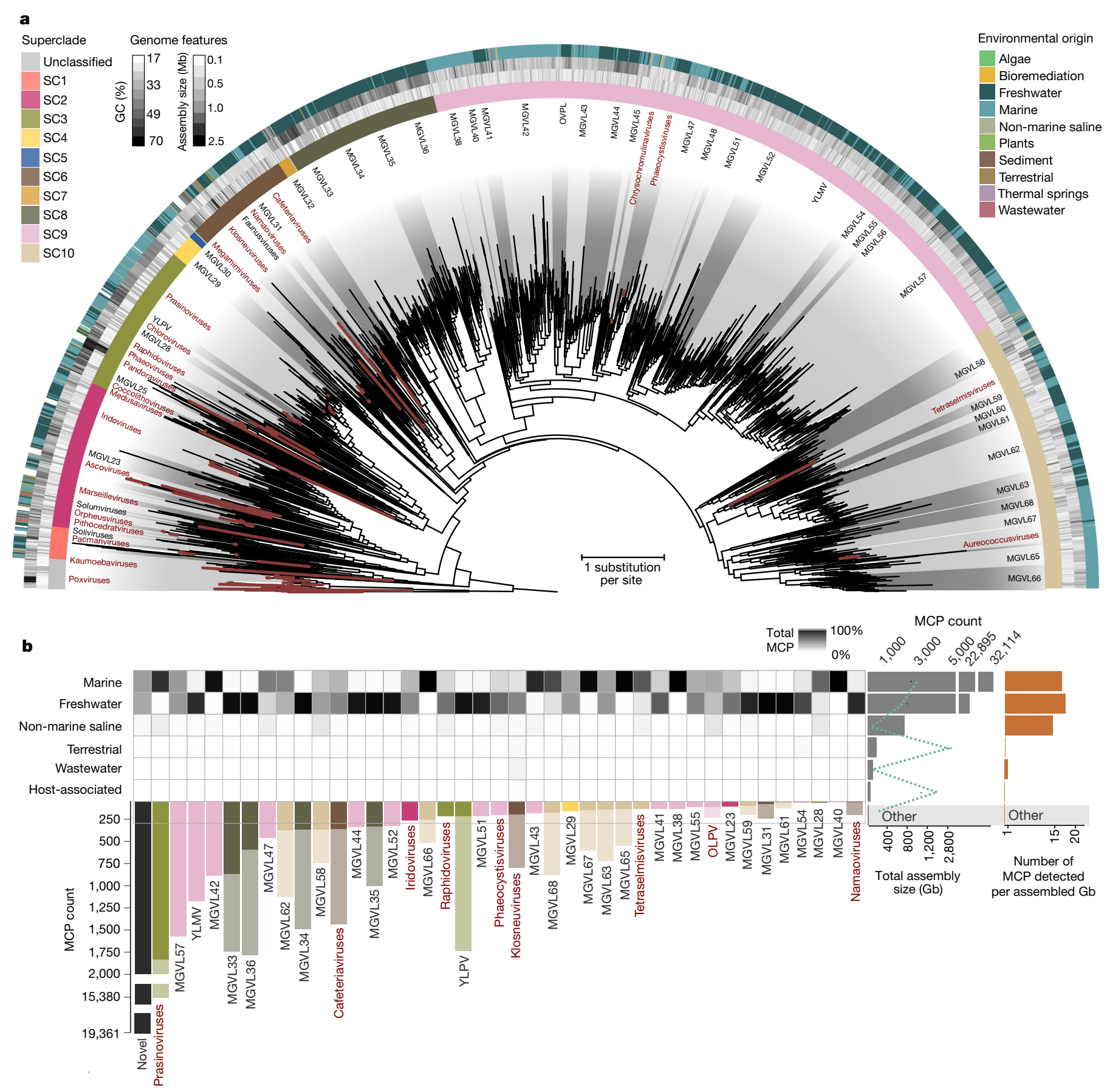

Fig. 1 Metagenomic expansion of the NCLDV diversity. a, Maximumlikelihood phylogenetic tree of the NCLDV inferred from a concatenated protein alignment of five core $\mathrm{NCVOG}^{16}$. Branches in dark red represent published genomes and branches in black represent GVMAGs generated in this study. Shades of grey indicate boundaries of genus- and subfamily-level clades; previously described lineages are labelled. Tree annotations from inside to the outside: (1) superclade (SC), (2) GC content, (3) assembly size and (4) environmental origin. $\mathbf{b}$, Distribution of NCLDV lineages across different habitats. The bars adjacent to the heat map show the total number of detected
MCPs per habitat (facing to the right) and per lineage (facing downwards) as total count (total bar length) and corrected count on the basis of the average copy number of MCPs in the respective lineage (darker shaded bar length). The plot includes only lineages for which at least 100 MCPs could be detected. NCLDV lineages with available virus isolates are indicated in red. The turquoise dashed line indicates the total size of the metagenome assemblies that were screened in this analysis. Bars on the far right indicate, for each environment, the number of detected MCPs per assembled gigabase (Gb).

indicated by a low number of shared protein families compared with the total number of protein families (Extended Data Fig. 5c). MGVL27, medusaviruses, sylvanviruses and MGVL24 represented the viral lineages with the highest genome novelty; for these lineages, on average, less than $15 \%$ of proteins showed similarity to known NCLDV proteins (Extended Data Fig. 6). Notably, clades that had been predominantly
Data Fig. 5b). MGVL57, MGVL58, YLMVs and klosneuviruses were the most-diverse lineages on the basis of their overall gene content, as 


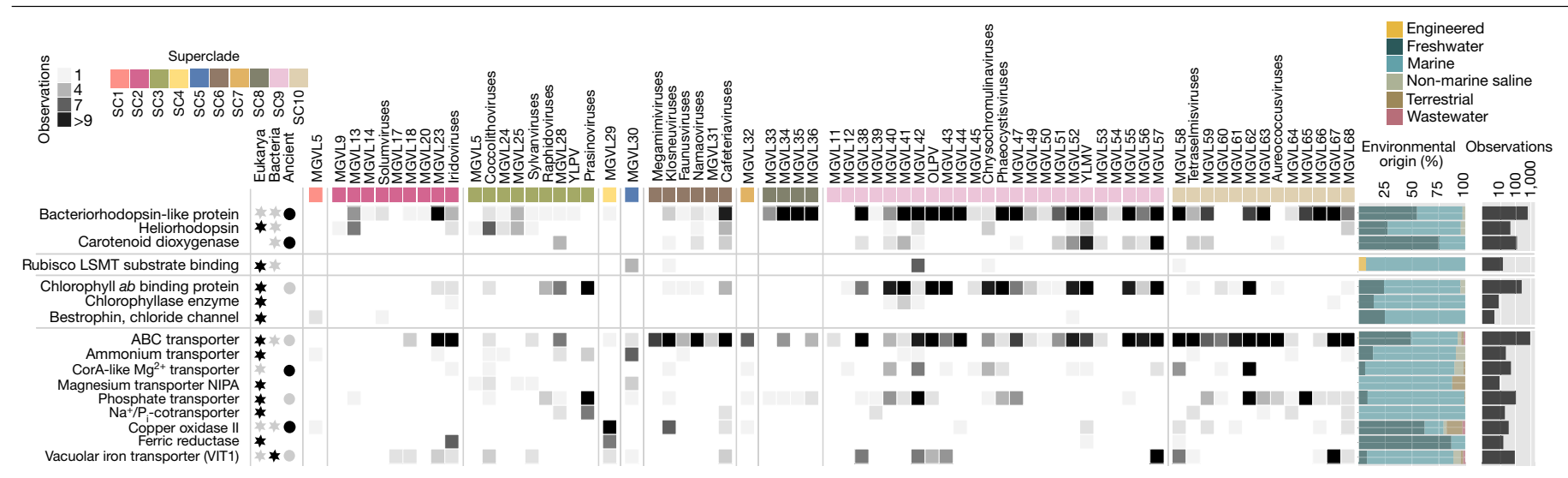

Fig. 2 |NCLDV coding potential and proteins that are probably involved in metabolic host reprogramming. Copy numbers of selected $\mathrm{Pfam}$ domains with potential roles as light-driven proton pumps, in carbon fixation, in photosynthesis and in diverse substrate transport processes. Filled stars and circles specify observed modes of transmission of the respective Pfamdomain-containing proteins. Stars represent recent HGTs from either eukaryotes or bacteria; circles indicate vertical transmission after ancient HGT or gene birth in the NCLDV; a darker colour indicates the predominantly observed mode of transmission (five or more events). The stacked bars on the right side of the heat map show, for each observed protein domain, the proportional distribution across different habitat types. Bars on the far right indicate the total number of observations for each protein domain. sampled in the past with several viral isolate genomes sequenced, such as marseilleviruses, poxviruses, pandoraviruses and faustoviruses, were nearly absent in the environmental microbiome data. This finding indicates that these viruses or their hosts have comparably low abundances in the samples analysed our dataset. It also suggests that there is a skew in the isolation and co-cultivation efforts of giant viruses using selected non-native hosts in laboratory setup ${ }^{18-20}$. Large-scale, cultivation-independent genome-resolved metagenomics alleviates such bias and provides a more-global snapshot of diversity and the spatial distribution of NCLDVs in their natural habitats.

To further deepen our understanding of the environmental distribution patterns of the NCLDVs, we performed a survey of the major capsid protein (MCP) across all public metagenomic datasets. We identified more than 58,000 copies of this protein, of which $67 \%$ could be assigned to viral lineages (Fig. 1b). Among the most-commonly found lineages were prasinoviruses, MGVL57 and YLMV with more than 1,000 occurrences each. At the same time, only a few MCPs (less than 100) were detected in viruses that have repeatedly been isolated in co-cultivation with amoebae, such as megamimiviruses, marseilleviruses and faustoviruses $^{18-20}$. In our environmental survey, MCPs were predominantly found in marine (around 55\%) and freshwater (about 40\%) and-to a much lesser extent-in terrestrial (less than $1 \%$ ) environments. Some NCLDV lineages occurred solely in either freshwater (YLMV, MGVL33 and MGVL36) or marine (prasinoviruses, MGVL42 and MGVL66) systems, whereas members of other lineages were found in both-or in an even-wider range of-environments (such as klosneuviruses, which were found in freshwater, marine, non-marine saline, terrestrial, wastewater and host-associated ecosystems). Large and giant viruses could also be detected in hydrothermal vents and thermal springs; however, comparably few MCPs were present in these habitats (Fig. 1b). Projecting the distribution of NCLDVs onto a global scale makes their ubiquitous nature apparent (Extended Data Fig. 7). These viruses can be found almost anywhere with many different lineages often co-occurring in close proximity to each other, suggesting that their discovery is chiefly limited by sampling effort.

Considering the ubiquitous prevalence of large and giant viruses, we aimed to investigate the potential influences that these viruses have on their hosts. The detrimental effect of viral infections on their eukaryotic hosts are well-known'; however, a few recent studies have shown that NCLDVs might also complement the metabolism of their host, for example, by encoding transporters that take up nutrients, such as nitrogen, or fermentation genes ${ }^{21,22}$. Expanding these initial findings, our data showed that diverse lineages across all NCLDV superclades encoded enzymes with potential roles in photosynthesis, diverse substrate transport processes, light-driven proton pumps and retinal pigments (Fig. 2). Maps of the presence, absence and prevalence of these genes revealed lineage- and environment-specific patterns. Most-commonly observed across a wide-range of habitats were ABC transporters, chlorophyll $a b$-binding proteins and bacteriorhodopsin-like proteins (Fig. 2, Supplementary Note 2 and Supplementary Table 5). Transporters for ammonium, magnesium and phosphate, which are likely to be of importance for hosts in oligotrophic environments such as the surface ocean, were predominantly found in marine viruses. Enzymes such as ferric reductases and multicopper oxidases-which facilitate the uptake of iron ${ }^{23,24}$, an essential trace element that is often growth-limiting, especially in photosynthetic organisms ${ }^{25}$ - were encoded in GVMAGs sampled across different habitats. This wealth of virus-encoded genes with roles in energy generation and nutrient acquisition has far-reaching implications for ecosystem dynamics. Metabolic reprogramming refers to a common phenomenon in which bacterial viruses obtain genes from their hosts and maintain them to support host metabolism ${ }^{26}$. Our results illustrate that in a similar manner, NCLDV-mediated host reprogramming is probably an important strategy to increase viral fecundity and at the same time render a short-term competitive advantage of infected eukaryotic host cells, especially under nutrient-limited conditions.

In agreement with previous studies ${ }^{27-30}$, many of the identified viral genes with predicted effects on host cell processes were probably acquired from their hosts through horizontal gene transfer (HGT) (Fig. 2 and Extended Data Fig. 8). Other genes were present across different viral lineages and superclades, suggesting ancient transfer followed by vertical inheritance during the course of NCLDV evolution or the origin of the respective gene in a common ancestor of this group of viruses. A notable example is the group of rhodopsin-like domain-containing proteins, which we found in 555 of the GVMAGs. Type-1 rhodopsins in algae-infecting phycodnaviruses and in viruses of heterotrophic choanoflagellates have been reported in previous studies and comprise viral rhodopsin groups I and II ${ }^{10,31,32}$. However, in light of our extended sampling of NCLDV genomes, it becomes evident that NCLDVs encoded more-diverse rhodopsins than described (Extended Data Fig. 8), which comprise approximately one quarter of the total known diversity of rhodopsins and include proteins from all publicly available metagenomes (Extended Data Fig. 9). Notably, the phylogeny 


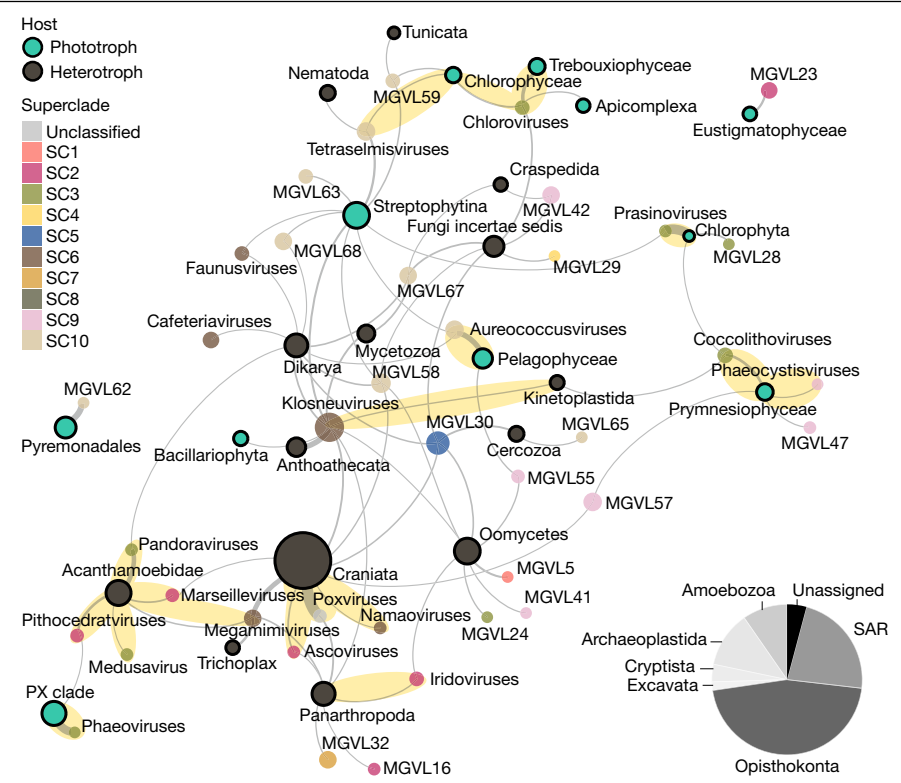

Fig. 3 HGT between NCLDV and their putative eukaryotic hosts. Undirected HGT network with nodes that represent previously described viral lineages and MGVLs, coloured on the basis of NCLDV superclade affiliation, with names above the node and their putative hosts (highlighted in black with names below the node, coloured on the basis of lifestyle); edges are weighted on the basis of the number of detected transfers. Connections comprising at least four transfers are shown. Experimentally verified virus-host associations are highlighted in yellow with names in bold. The proportion of HGT candidates assigned to hosts from different major eukaryotic lineages is shown as a pie chart.

of the viral rhodopsins from all NCLDV superclades exhibits a strongly supported monophyletic signal, which implies that this gene might represent an ancestral trait of the NCLDV that was subsequently lost in some lineages. In addition to viral rhodopsin group I and II, additional NCLDV rhodopsins branch closely to their cellular counterparts and have probably been acquired by HGT from different hosts (Extended Data Fig. 8). In a similar manner, putative NCLDV heliorhodopsins were found intertwined with their homologues in the algae Chrysochromulina and Micromonas (Extended Data Fig. 8). In addition to the rhodopsins, our dataset contained 119 GVMAGs that encoded carotenoid oxygenases, which potentially modulate light-harvesting capacity or synthesize bioactive compounds ${ }^{33}$. It is conceivable that some of the NCLDV rhodopsins function in conjunction with the carotenoid oxygenases and have important roles in modulating host-cell processes; for example, by acting as light-driven proton pumps, as photoreceptors in host phototactic motility or as photoprotectants $\mathrm{s}^{10,34,35}$-each of these functions lead to metabolic advantages of infected populations.

Uptake of host genes is a common mechanism in the evolution of $\mathrm{NCLDVs}^{2,11,30,36}$. Using HGT analyses, we assigned putative hosts to different NCLDV lineages. Analysis of 2,040 genes that have probably undergone HGT provided linkage information for 50 viral lineages to 32 groups of putative eukaryotic hosts (Fig. 3 and Supplementary Table 6). Notably, 17 out of 23 viral lineages that contained genomes from isolated viruses could be connected through HGT to their experimentally verified native hosts, such as most algae-infecting viruses and metazoa-infecting ascoviruses, namaoviruses and poxviruses, as well as connecting klosneuviruses to Kinetoplastida ${ }^{37,38}$. Our analysis further confirmed Acanthamoeba as a host of pandoraviruses, pithocedratviruses, medusaviruses, marseilleviruses and megamimiviruses. Notably, megamimiviruses, which have exclusively been obtained through co-cultivation with amoebae, showed not only HGT with this host but were linked even more strongly to multicellular animals. The best-connected NCLDV lineage was the klosneuviruses, a viral subfamily mainly known from metagenomic studies, ${ }^{9,1,12,39}$. Our HGT network revealed that klosneuviruses have a diverse putative host range of mainly heterotrophs, including Anthoathecata-to which it showed the strongest connection-as well as fungi and arthropods, and different protists, including slime moulds. By contrast, Oomycetes, Dikarya, fungi incertae sedis and Streptophytina emerged as putative hosts for the greatest number of different NCLDV lineages, despite the lack of isolation of NCLDVs from any of these organisms. With predicted hosts in Opisthokonta, Amoebozoa, Excavata, Archaeoplastida, Cryptista and the Stramenopila, Alveolata, Rhizaria (SAR) supergroup, our results suggest that members of the NCLDV might be able to infect most major eukaryotic lineages ${ }^{40}$ (Fig. 3). This is consistent with previous reports based on eukaryotic genome data ${ }^{27}$ and experimental data showing that large and giant viruses infect marine arrow worms ${ }^{41}$, epithelial cells in fish gills ${ }^{38}$ and potentially also corals and sponges ${ }^{42}$. Of note, our analysis did not reveal linkage to human hosts. We expect that with improved sampling of host genomes-particularly genomes of underexplored protists and algae-host linkage through HGT will yield an even more comprehensive picture of the host range and evolutionary histories of NCLDVs.

Overall, we leveraged the availability of metagenomic datagenerated by the global sampling efforts of a community of scientists to expand our insights into the diversity, host metabolic complementation and putative host range of large and giant viruses. NCLDV infections probably occur in all major eukaryotic lineages, with repercussions for many of Earth's major biogeochemical processes. Our data and findings represent a solid foundation and expansive resource for future giant-virus research efforts to deepen our understanding of the evolutionary and ecological bearings of these viral giants.

\section{Online content}

Any methods, additional references, Nature Research reporting summaries, source data, extended data, supplementary information, acknowledgements, peer review information; details of author contributions and competing interests; and statements of data and code availability are available at https://doi.org/10.1038/s41586-020-1957-x.

1. Abergel, C., Legendre, M. \& Claverie, J.-M. The rapidly expanding universe of giant viruses: mimivirus, pandoravirus, pithovirus and mollivirus. FEMS Microbiol. Rev. 39, 779-796 (2015).

2. Koonin, E. V. \& Yutin, N. Evolution of the large nucleocytoplasmic DNA viruses of eukaryotes and convergent origins of viral gigantism. Adv. Virus Res. 103, 167-202 (2019).

3. Abrahão, J. et al. Tailed giant Tupanvirus possesses the most complete translational apparatus of the known virosphere. Nat. Commun. 9, 749 (2018).

4. Fischer, M. G. Giant viruses come of age. Curr. Opin. Microbiol. 31, 50-57 (2016).

5. Mihara, T. et al. Taxon richness of "Megaviridae" exceeds those of Bacteria and Archaea in the ocean. Microbes Environ. 33, 162-171 (2018).

6. Hingamp, P. et al. Exploring nucleo-cytoplasmic large DNA viruses in Tara Oceans microbial metagenomes. ISME J. 7, 1678-1695 (2013).

7. Monier, A., Claverie, J.-M. \& Ogata, H. Taxonomic distribution of large DNA viruses in the sea. Genome Biol. 9, R106 (2008).

8. Wilson, W. H., Van Etten, J. L. \& Allen, M. J. The Phycodnaviridae: the story of how tiny giants rule the world. Curr. Top. Microbiol. Immunol. 328, 1-42 (2009).

9. Schulz, F. et al. Hidden diversity of soil giant viruses. Nat. Commun. 9, 4881 (2018).

10. Needham, D. M. et al. A distinct lineage of giant viruses brings a rhodopsin photosystem to unicellular marine predators. Proc. Natl Acad. Sci. USA 116, 20574-20583 (2019).

11. Schulz, F. et al. Giant viruses with an expanded complement of translation system components. Science 356, 82-85 (2017).

12. Bäckström, D. et al. Virus genomes from deep sea sediments expand the ocean megavirome and support independent origins of viral gigantism. mBio 10, e02497-18 (2019).

13. Andreani, J., Verneau, J., Raoult, D., Levasseur, A. \& La Scola, B. Deciphering viral presences: two novel partial giant viruses detected in marine metagenome and in a mine drainage metagenome. Virol. J. 15, 66 (2018).

14. Wilson, W. H. et al. Genomic exploration of individual giant ocean viruses. ISME J. 11, 1736-1745 (2017).

15. Chen, I. A. et al. IMG/M v.5.0: an integrated data management and comparative analysis system for microbial genomes and microbiomes. Nucleic Acids Res. 47, D666-D677 (2019).

16. Yutin, N., Wolf, Y. I., Raoult, D. \& Koonin, E. V. Eukaryotic large nucleo-cytoplasmic DNA viruses: clusters of orthologous genes and reconstruction of viral genome evolution. Virol. J. 6, 223 (2009).

17. Roux, S. et al. Minimum Information about an Uncultivated Virus Genome (MIUViG). Nat. Biotechnol. 37, 29-37 (2019). 


\section{Article}

18. Aherfi, S., Colson, P., La Scola, B. \& Raoult, D. Giant viruses of amoebas: an update. Front. Microbiol. 7, 349 (2016).

19. Boughalmi, M. et al. High-throughput isolation of giant viruses of the Mimiviridae and Marseilleviridae families in the Tunisian environment. Environ. Microbiol. 15, 2000-2007 (2013)

20. Reteno, D. G. et al. Faustovirus, an asfarvirus-related new lineage of giant viruses infecting amoebae. J. Virol. 89, 6585-6594 (2015).

21. Monier, A. et al. Host-derived viral transporter protein for nitrogen uptake in infected marine phytoplankton. Proc. Natl Acad. Sci. USA 114, E7489-E7498 (2017).

22. Schvarcz, C. R. \& Steward, G. F. A giant virus infecting green algae encodes key fermentation genes. Virology 518, 423-433 (2018).

23. Saikia, S., Oliveira, D., Hu, G. \& Kronstad, J. Role of ferric reductases in iron acquisition and virulence in the fungal pathogen Cryptococcus neoformans. Infect. Immun. 82, 839-850 (2014).

24. Herbik, A., Bölling, C. \& Buckhout, T. J. The involvement of a multicopper oxidase in iron uptake by the green algae Chlamydomonas reinhardtii. Plant Physiol. 130, 20392048 (2002).

25. Morrissey, J. \& Bowler, C. Iron utilization in marine cyanobacteria and eukaryotic algae. Front. Microbiol. 3, 43 (2012).

26. Hurwitz, B. L., Hallam, S. J. \& Sullivan, M. B. Metabolic reprogramming by viruses in the sunlit and dark ocean. Genome Biol. 14, R123 (2013).

27. Gallot-Lavallée, L. \& Blanc, G. A glimpse of nucleo-cytoplasmic large DNA virus biodiversity through the eukaryotic genomics window. Viruses $\mathbf{9}, 17$ (2017).

28. Finke, J. F., Winget, D. M., Chan, A. M. \& Suttle, C. A. Variation in the genetic repertoire of viruses infecting Micromonas pusilla reflects horizontal gene transfer and links to their environmental distribution. Viruses $\mathbf{9}, 116$ (2017).

29. Maumus, F. \& Blanc, G. Study of gene trafficking between Acanthamoeba and giant viruses suggests an undiscovered family of amoeba-infecting viruses. Genome Biol. Evol. 8, 3351-3363 (2016)

30. Filée, J. \& Chandler, M. Gene exchange and the origin of giant viruses. Intervirology $\mathbf{5 3}$ 354-361 (2010).

31. Philosof, A. \& Béjà, O. Bacterial, archaeal and viral-like rhodopsins from the Red Sea. Environ. Microbiol. Rep. 5, 475-482 (2013).

32. Yutin, N. \& Koonin, E. V. Proteorhodopsin genes in giant viruses. Biol. Direct 7, 34 (2012).

33. Ahrazem, O., Gómez-Gómez, L., Rodrigo, M. J., Avalos, J. \& Limón, M. C. Carotenoid cleavage oxygenases from microbes and photosynthetic organisms: features and functions. Int. J. Mol. Sci. 17, 1781 (2016).
34. Ernst, O. P. et al. Microbial and animal rhodopsins: structures, functions, and molecular mechanisms. Chem. Rev. 114, 126-163 (2014).

35. Sineshchekov, O. A., Jung, K.-H. \& Spudich, J. L. Two rhodopsins mediate phototaxis to low- and high-intensity light in Chlamydomonas reinhardtii. Proc. Natl Acad. Sci. USA 99 8689-8694 (2002)

36. Moreira, D. \& Brochier-Armanet, C. Giant viruses, giant chimeras: the multiple evolutionary histories of Mimivirus genes. BMC Evol. Biol. 8, 12 (2008).

37. Deeg, C. M. Chow, C. T. \& Suttle, C. A. The kinetoplastid-infecting Bodo saltans virus (BsV), a window into the most abundant giant viruses in the sea. eLife 7, e33014 (2018).

38. Clouthier, S., Anderson, E., Kurath, G. \& Breyta, R. Molecular systematics of sturgeon nucleocytoplasmic large DNA viruses. Mol. Phylogenet. Evol. 128, 26-37 (2018)

39. Stough, J. M. A. et al. Diversity of active viral infections within the Sphagnum microbiome. Appl. Environ. Microbiol. 84, e01124-18 (2018).

40. Adl, S. M. et al. The revised classification of eukaryotes. J. Eukaryot. Microbiol. 59, 429514 (2012).

41. Shinn, G. L. \& Bullard, B. L. Ultrastructure of Meelsvirus: a nuclear virus of arrow worms (phylum Chaetognatha) producing giant "tailed" virions. PLoS ONE 13, e0203282 (2018)

42. Claverie, J.-M. et al. Mimivirus and Mimiviridae: giant viruses with an increasing number of potential hosts, including corals and sponges. J. Invertebr. Pathol. 101 172-180 (2009)

Publisher's note Springer Nature remains neutral with regard to jurisdictional claims in published maps and institutional affiliations.

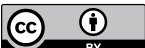

Open Access This article is licensed under a Creative Commons Attribution 4.0 International License, which permits use, sharing, adaptation, distribution and reproduction in any medium or format, as long as you give appropriate credit to the original author(s) and the source, provide a link to the Creative Commons license, and indicate if changes were made. The images or other third party material in this article are included in the article's Creative Commons license, unless indicated otherwise in a credit line to the material. If material is not included in the article's Creative Commons license and your intended use is not permitted by statutory regulation or exceeds the permitted use, you will need to obtain permission directly from the copyright holder. To view a copy of this license, visit http://creativecommons.org/licenses/by/4.0/.

(c) The Author(s) 2020 


\section{Methods}

\section{Generation of models to detect NCLDV proteins}

Initial hidden Markov models (HMMs) for the MCPs were built from a multiple sequence alignment of published NCLDV MCPs and subsequently updated on the basis of extracted metagenomic NCLDV MCP sequences. We screened around 537 million proteins encoded on about 45.1 million contigs with a length greater than $5 \mathrm{~kb}$ available in 8,535 public metagenomes in IMG $/ \mathrm{M}^{43}$ (June 2018) for contigs that encode the NCLDV MCP using a version of hmmsearch (v.3.1b2, http://hmmer.org/) that is optimized ${ }^{44}$ for the supercomputer Cori, with a set of models for the NCLDV MCP (https://bitbucket.org/berkeleylab/mtg-gv-exp/) and an $E$-value cut-off of $1 \times 10^{-10}$. The $1,003,222$ proteins found on the 77,701 contigs with hits for MCPs were then clustered with CD-hit ${ }^{45}$ at a sequence similarity of $99 \%$ to remove nearly identical and identical proteins. This resulted in 524,161 clusters and singletons. The cluster representatives were used to infer protein families using orthofinder (v.2.27) with default settings and the -diamond flag ${ }^{46,47}$. Multiple sequence alignments were built with mafft ${ }^{48}(v .7 .294 b)$ for protein families that included at least 10 members and corresponding HMM models were obtained with hmmbuild (v.3.1b2, http://hmmer. org/). This led to a total of 7,182 HMMs that can detect NCDLV proteins that were then tested against all public genomes in $\mathrm{IMG} / \mathrm{M}^{43}$ (June 2018). Models that gave rise to hits above an $E$-value cut-off of $1 \times 10^{-10}$ in more than 10 reference genomes were removed. The resulting 5,064 models were then used for targeted binning of NCLDV metagenome contigs.

\section{Identification of NCLDV-specific genome features and design of an automatic classifier}

A set of representative genomes of bacteria, archaea, eukaryotes and non-NCLDV viruses was gathered from the IMG/M database $\mathrm{e}^{43}$ (June 2018) and combined with NCLDV genomes assembled from metagenomes and protist genomes downloaded from NCBI GenBank to identify NCLDV-specific genome features. Genes were predicted for these genomes using Prodigal ${ }^{49}$ (v.2.6.3; February, 2016) in both 'regular' mode (default parameters) and with the option ' $-n$ ' activated, which forces a full motif scan. For genomes of less than $100 \mathrm{~kb}$, the option '-p meta' was used to apply precalculated training files rather than training the gene predictor from the genome, as recommended by the tool documentation. Next, a set of different metrics was calculated for each genome on the basis of the genes predicted with a confidence of $\geq 90$ and score of $\geq 50$. These included gene density (number of genes predicted on average per $10 \mathrm{~kb}$ of genome), coding density (number of bp predicted as part of a coding sequence per $10 \mathrm{~kb}$ of genome), spacer length (average length of the spacer between the predicted ribosomal binding site (RBS)), predicted start codon for genes in which a putative RBS was detected and RBS motif profile (the proportion of each type of RBS predicted in the genome, see below).

For the RBS motif profile, motifs were predicted using the full motif scan option of prodigal (see above). Notably, some of these motifs may not represent true RBSs, but are instead other conserved motifs (including transcription-related motifs) found upstream of start codons in these different genomes. These motifs were grouped into 11 categories as follows: (1) 'None' for cases in which prodigal did not predict a RBS; (2) 'SD_Canonical' for different variations of the canonical AGGAGG Shine-Dalgarno sequence (for example, AGGAG, AGxAG, GAGGA, as well as motifs identified by Prodigal as '3Base_5BMM' or '4Base_6BMM'); (3) 'SD_Bacteroidetes' for variations of the motif predicted typically from Bacteroidetes genomes (TA $\{2,5\} T\{0,1\}$ : T followed by $2-5 \mathrm{As}$, and with sometimes a terminal T); (4) 'Other_GA' for motifs that include ' $G A$ ' patterns but that are different from the canonical Shine-Dalgarno sequence, for example, GAGGGA, typically identified in a few archaeal and bacterial genomes; (5) 'TATATA_3.6' for variations of the motif typically detected in NCLDV, that is, a motif of 3-6 bp with alternating Ts and As (TAT, ATAT, TATA, TATAT, and so on); (6) 'OnlyA' for motifs exclusively composed of As not already included in a previous group, for example, AAAAA, most often found in Bacteroidetes; (7) 'OnlyT' for motifs exclusively composed of Ts not already included in a previous group, for example, TTTTT, found at a low frequency in some archaeal genomes; (8) 'DoubleA' for motifs with two consecutive As not already included in a previous group, for example, AAAAC, most often found in Bacteroidetes and bacteria from the candidate phyla radiation (CPR) group; (9) 'DoubleT' for motifs with two consecutive Ts not already included in a previous group, for example, TACTT, found at a low frequency in plants, Bacteroidetes and NCLDV; (10) 'NoA' for motifs without any As and not included in a previous group, for example, TCTCG, found in some archaeal genomes; and (11) 'Other' for motifs that did not fit into any of these categories.

Representative genomes were then grouped on the basis of the frequency of each motif type through hierarchical clustering ( $R$ function 'hclust'). This enabled the delineation of 12 genome groups on the basis of taxonomy (at the kingdom or domain ranks) and motif profile (Extended Data Fig. 2). Two types of random-forest classifiers were then built on the basis of the 14 features (11 motifs, gene density, coding density and average spacer length, see above): one for which the category to be predicted was binary (that is, 'Virus_NCLDV' versus 'Other') and one for which the category to be predicted was the set of genome groups based on predicted RBS motifs ('NCLDV (non-pandoraviruses)', 'animal and plants', 'protists \& fungi', 'canonical bacteria and archaea', 'bacteroidetes-like', 'bacteria (CPR)', 'atypical bacteria', 'atypical archaea', 'plasmids' and 'other viruses', which include pandoraviruses). The 14 features were evaluated on the whole genomes, as well as on fragments of $20 \mathrm{~kb}$ and $10 \mathrm{~kb}$ selected randomly along the genomes. These random fragments were used to train a classifier on input sequences more comparable to metagenome assemblies, which most often represent short genome fragments of a few kb. For these fragments, Prodigal was run with the '-p meta' option and default parameters otherwise ${ }^{50}$, that is, without a full motif scan, as these sequences are typically too short to identify de novo RBS motifs. Animal and plant genomes were not included in this analysis as these are highly unlikely to be assembled from metagenomes. All classifiers were built using R library randomforest and included 2,000 trees, with default parameters otherwise, and 10 -fold cross-validation was performed to evaluate the classifier accuracy. The probability 'prob' of NCLDV origin was used as a prediction score to evaluate the classifiers and was then applied to metagenome assemblies. Because the input dataset is easily skewed towards bacterial and archaeal genomes, specificity and sensitivity were evaluated separately for each group of genome (Extended Data Fig. 2c). Statistical tests were performed in R using the package stats (Kolmogorov-Smirnov test) ${ }^{51}$ and effsize $(\text { Cohen's effect size })^{52}$.

\section{MAGs from non-targeted binning of IMG genomes}

Complementary to the targeted binning of NCLDV contigs, we performed genome binning of public metagenomes in IMG/M (assessed June 2018) $)^{15}$ with MetaBAT (v.0.32.4) ${ }^{53}$ in the 'superspecific' mode, using read coverage information, if available in IMG, and a minimum contig length of $5 \mathrm{~kb}$. Resulting MAGs were then checked for quality using CheckM (v.1.0.7) $)^{54}$. Genome bins with completeness $<50 \%$ were labelled as low quality according to the 'minimum information for a MAG' (MIMAG) standards ${ }^{55}$.

\section{Targeted binning of putative NCLDV metagenome contigs}

The 5,064 NCLDV-specific models were used for hmmsearch (v.3.1b2, http://hmmer.org/) on the initial set of around 537 million proteins encoded on about 45 million contigs with a length greater than $5 \mathrm{~kb}$ with an $E$-value cut-off of $1 \times 10^{-10}$ (Extended Data Fig. 1 ). In addition to the screening of the metagenomic contigs with NCLDV-specific models, we also used an automatic classifier using gene density and RBS motifs 
(see above). On the basis of the output of the automatic classifier, a score was assigned to each contig: a score of 2 if Ratio_TATATA_36 $>0.3$ or Pred_simple_NCLDV_score $>0.3$ and the prediction result was 'Virus_NCLDV', a score of 1 if Ratio_TATATA_36 $>0.3$ or Pred_simple_ NCLDV_score $>0.1$ or the prediction result was 'Virus_NCLDV', otherwise a score of 0 . On the basis of the cross-validation of the classifier, these parameters were chosen to maximize sensitivity while retaining enough specificity. The resulting set of around 1.2 million contigs with an RBS score of at least 1 and/or at least $20 \%$ of encoded genes (1 out of 5) with hits to the NCLDV models were subject to metagenomic binning as follows: for each metagenome, putative NCLDV contigs were extracted and binning performed with $\operatorname{MetaBAT}^{56}(\mathrm{v} .2)$ and contig read coverage information was used as input in case it was available in $\mathrm{IMG}^{43}$. The targeted binning approach gave rise to around 72,000 putative NCLDV MAGs.

\section{Filtering of GVMAGs}

Contigs with a length of less than $5 \mathrm{~kb}$ were removed from GVMAGs. Filtering was performed on the basis of the copy number of NCVOGs $^{16}$ (Supplementary Tables 2, 3). GVMAGs were removed when they encoded more than 20 copies of NCVOG0023, 4 copies of NCVOG0038, 12 copies of NCVOG0076, 7 copies of NCVOG0249 or 4 copies of NCVOG0262. On the basis of the copy numbers of 16 conserved NCOVGs (NCVOG0035, NCVOG0036, NCVOG0038, NCVOG0052, NCVOG0059, NCVOG0211, NCVOG0249, NCVOG0256, NCVOG0262, NCVOG1060, NCVOG1088, NCVOG1115, NCVOG1117, NCVOG1122, NCVOG1127 and NCVOG1192), which are usually present at low copy numbers across all published NCLDV genomes, a duplication ratio was calculated as follows. The total number of copies of the 16 NCVOGs in the respective GVMAG was divided by the total number of unique observations of the 16 NCVOGs. GVMAGs with a duplication ratio higher than three were excluded from the dataset. We then used Diamond BLASTp ${ }^{47}$ against the NCBI non-redundant (nr) database (August 2018) and assigned a taxonomic affiliation on the basis of best BLASTp hits against Archaea, Bacteria, Eukaryota, phages or other viruses (including NCLDVs) to proteins using an $E$-value cutoff of $1 \times 10^{-5}$. Best hits of query proteins to proteins derived from MAGs from the Tara Mediterranean metagenome binning survey ${ }^{57}$ were disregarded owing to the high number of misclassified genomes in this dataset. Proteins without a hit in the NCBI nr database were labelled as 'Unknown'. We then applied filters to remove contigs from GVMAGs on the basis of the distribution of taxonomic affiliation of best blast hits (Supplementary Table 7). Finally, alignments were built with mafft ${ }^{48}$ (v.7.294b) for NCVOG0023, NCVOG0038, NCVOG0076, NCVOG0249 and NCVOG0262. Positions with $90 \%$ or more gaps were removed from the alignments with trimal ${ }^{58}$ (v.1.4). Protein alignments were concatenated and a species tree constructed with IQ-tree ${ }^{59}$ ( LG + F + R8, v.1.6.10). The phylogenetic tree was then manually inspected and for each clade outliers were removed on the basis of the presence, absence and copy numbers of 20 conserved $\mathrm{NCVOGs}^{16}$, duplication factor (see above), coding density, GC content and genome size. In addition, GVMAGs that represented singletons on long branches were manually removed. The filtered dataset was then clustered together with all available NCLDV reference genomes (December 2018) using average nucleotide identities of greater than $95 \%$ and an alignment fraction of at least $50 \%$ with FastANI $^{60}$ (v.1.1). For each $95 \%$ average nucleotide identity cluster the $6 \mathrm{NCVOGs}^{16}$ with the on-average longest amino acid sequences (NCVOG0022, NCVOG0023, NCVOG0038, NCVOG0059, NCVOG0256 and NCVOG1117) were subjected to a within-cluster all-versus-all BLASTp. GVMAGs that had any full-length $100 \%$ identity hits between any of these maker proteins to other cluster members were removed from the dataset as potential duplicates. Duplicate GVMAGs originating from the conventional binning approach were removed first and GVMAGs with the largest assembly size were retained.

\section{GVMAG quality on the basis of estimated completeness and contamination}

Estimation of the quality of MAGs is critical for their interpretation and use in downstream applications. Standards exist for bacterial and archaeal MAGs that have proposed a three-tier classification (high, medium or low quality) based on estimated genome completeness and contamination ${ }^{55}$. These completeness and contamination metrics are typically calculated on the basis of a set of universal single-copy marker genes. A set of conserved genes in the NCLDV are the NCVOGs ${ }^{16}$, of which a subset has been shown to be probably vertically inherited ${ }^{16}$ (NCVOG20, Supplementary Table 2). We calculated for each superclade the average number of NCVOG20 present either as a single copy or as multiple copies (Supplementary Table 3). We then compared the number of observed single- and multicopy NCVOG20 in every GVMAG to the mean number of observations in the respective superclade. Considering the high genome plasticity of $\mathrm{NCLDVs}^{2,61}$, we tolerated a deviation from the mean by a factor of 1.2 , which was considered low contamination, and a factor of 2 was considered medium contamination (Extended Data Fig. 4 and Supplementary Table 4). Higher deviations from the superclade mean were potentially caused by a non-clonal composition of the GVMAG; these were, as a consequence, considered to be of high contamination. We also estimated completeness on the basis of the presence of the NCVOG 20 compared with other members of the respective superclade. The presence of $90 \%$ or more of the NCVOG20 compared with the superclade mean resulted in a classification as high quality in terms of completeness. If at least $50 \%$ of NCVOG20 were present in a GVMAG then the respective GVMAG was classified as medium quality in terms of estimated completeness, or low if less than 50\% of NCVOG20 were present (Extended Data Fig. 4 and Supplementary Table 4). The final GVMAG quality was determined on the basis of a combination of contamination and completeness (Supplementary Table 8). Additional criteria to assign GVMAGs to the high-quality category were the presence of no more than 30 contigs, a minimum assembly size of $100 \mathrm{~kb}$ and the presence of at least one contig with a length greater than $30 \mathrm{~kb}$. To assign a GVMAG to the mediumquality category were the presence no more than 50 contigs, a minimum assembly size of $100 \mathrm{~kb}$ and the presence of at least one contig with a length greater than $15 \mathrm{~kb}$.

\section{Annotation of GVMAGs}

Gene calling was performed with GeneMarkS using the virus model ${ }^{62}$. For functional annotation proteins were subject to BLASTp against previously established NCVOGs ${ }^{16}$ and the NCBI nr database (May 2019) using Diamond (v.0.9.21) BLASTp ${ }^{47}$ with an $E$-value cut-off of $1.0 \times 10^{-5}$. In addition, protein domains were identified by pfam_scan.pl (v.1.6) against Pfam- $\mathrm{A}^{63}$ (v.29.0), and rRNAs and introns were identified with cmsearch using the Infernal package ${ }^{64}$ (v.1.1.1) against the Rfam database $^{65}$ (v.13.0). No rRNA genes were detected in the final set of GVMAGs. The eggNOG mapper ${ }^{66}$ (v.1.0.3) was used to assign functional categories to NCLDV proteins. Protein families were inferred with PorthoMCL ${ }^{67}$ (version of December 2018) with default settings.

\section{Survey of the NCLDV MCP}

We used hmmsearch (v.3.1b2, http://hmmer.org/) optimized for the supercomputer Cori ${ }^{44}$ to identify all copies of MCP encoded in the final set of GVMAGs and NCLDV reference genomes. Proteins were extracted and multiple sequence alignments were created with mafft ${ }^{48}$ (v.7.294b) for 74 NCLDV lineages with at least 5 copies of MCP. For each lineage-specific MCP alignment, we inferred models with hmmbuild (v.3.1b2, http://hmmer.org/). Using these models, the modified version of hmmsearch (v.3.1b2, http://hmmer.org/ $/{ }^{44}$ was used to identify all MCPs in the entire set of metagenomes (IMG/ $\mathrm{M}^{43}$, June 2018), MCPs with identical amino acid sequences were excluded as potential duplicates. A logistic-regression-based classifier (sklearn LogisticRegression, 
solver = 'lbfgs', multi_class = 'ovr') was trained for each NCLDV lineage taking into account the score distribution of all lineage MCPs hits against the entire set of lineage-specific MCP models. The accuracy of the classifier was 0.861. Unbinned metagenomic MCPs were assigned to NCLDV lineages if the classifier returned a probability greater than $50 \%$ (sklearn predict_proba), or as 'novel' if the probability was $50 \%$ or below. We then normalized the environmental MCP counts on the basis of the observed average copy number of MCP in GVMAGs and reference genomes in the respective lineage. Distribution of NCLDV lineages on the basis of MCPs was projected on a world map with Python 3/basemap on the basis of coordinates provided in IMG metagenomes ${ }^{43}$.

\section{NCLDV species tree}

To build a species tree of the extended NCLDV, viral genomes with at least three out of five core $\mathrm{NCVOG} \mathrm{s}^{16}$ were selected: DNA polymerase elongation subunit family B (NCVOG0038), D5-like helicase-primase (NCVOG0023), packaging ATPase (NCVOG0249), DNA or RNA helicases of superfamily II (NCVOG0076), and poxvirus late transcription factor VLTF3-like (NCVOG0262). The NCVOGs were identified with hmmsearch (version 3.1b2, http://hmmer.org/) using an $E$-value cut-off of $1 \times 10^{-10}$, extracted and aligned using mafft ${ }^{48}(v .7 .294 \mathrm{~b})$. Columns with less than $10 \%$ sequence information were removed from the alignment with trimal ${ }^{58}$. The species tree was then calculated on the basis of the concatenated alignment of all five proteins with IQ- $\operatorname{tre~}^{59}(\mathrm{v} \cdot 1.6 .10)$ with ultrafast bootstrap ${ }^{68}$ and $L G+F+R 8$ as suggested by model test as the best-fit substitution mode ${ }^{69}$. The percentage increase in phylogenetic diversity ${ }^{70}$ was calculated on the basis of the difference of the sum of branch lengths of the phylogenetic species trees of the NCLDV including the GVMAGs compared with a NCLDV species tree calculated from published NCLDV reference genomes ( $n=205$, no dereplication based on the average nucleotide identity) with IQ-tree as described above. Phylogenetic trees were visualized with $\mathrm{iTol}^{71}$ (v.5). Genus or subfamily level lineages were defined on the basis of their monophyly in the species tree and presence or absence pattern of conserved NCVOGs (Supplementary Table 4). If no viral isolates were present in the respective monophyletic clade we designated it MGVL. Neighbouring lineages with isolates and MGVLs were further combined under the working term superclade. Branch lengths separating clades differ based on the density of sampled viruses.

\section{Protein trees}

Target proteins were extracted from NCLDV genomes and used to query the NCBI nr database (June 2018) with Diamond BLASTp ${ }^{47}$. The top-50 hits per query were extracted, merged with queries, dereplicated on the basis of protein accession number and aligned with MAFFT (-linsi, v.7.294b) ${ }^{48}$, trimmed with trimal ${ }^{58}$ (removal of positions with more than $90 \%$ of gaps) and maximum-likelihood phylogenetic trees inferred with IQ-tree ${ }^{59}$ (multicore v.1.6.10) using ultrafast bootstrap ${ }^{68}$ and the model suggested by the model test feature implemented in IQ-tree ${ }^{69}$ based on Bayesian information criterion. Selected models are indicated in the legend of Extended Data Fig. 8. Owing to its size, the phylogenetic tree for $\mathrm{ABC}$ transporter was inferred with Fast Tree ${ }^{72}$ (v.2.1.10) LG and can be accessed at https://bitbucket.org/berkeleylab/mtg-gv-exp/. Phylogenetic trees were visualized with $\mathrm{iTol}^{71}$ (v.5). Information on functional genes including parent contigs is provided in Supplementary Table 5 .

\section{Virus-host linkage through HGT}

To generate a cellular nr database, all non-cellular sequences and sequences from the Tara Mediterranean genome study ${ }^{57}$ were removed from the NCBI nr database. All proteins in the NCLDV genomes were then subjected to Diamond BLASTp ${ }^{47}$ against the cellular nr database using an $E$-value cut-off of $1 \times 10^{-50}$, an alignment fraction of $50 \%$ and a minimum sequence identity of $50 \%$. Best blast hits within the same lineage were removed. Proteins that had a hit in cellular nr with a lower
$E$ value compared with hits in the NCLDV blast database were considered HGT candidates. The total number of best hits from lineage panproteomes against defined groups of Eukaryotes were then used as edge weights to build an HGT network. The network was created in Gephi (v.0.92) $)^{73}$ using a force layout and filtered at an edge weight of 2. Pfam annotations of HGT candidates were based on the most commonly detected domains and functional categories were assigned with the eggNOG Mapper (v.1.03) ${ }^{66}$. Information on HGT candidates including parent contigs is provided in Supplementary Table 6. The number of HGT linkages was limited by the available of reference genomes and the stringency applied.

\section{Reporting summary}

Further information on research design is available in the Nature Research Reporting Summary linked to this paper.

\section{Data availability}

All GVMAGs of estimated high and medium quality with an N50 of greater than $50 \mathrm{~kb}$ and estimated low contamination have been deposited at NCBI GenBank as MN738741-MN741037 under BioProject ID PRJNA588800. Nucleotide and protein sequences of GVMAGs can be directly downloaded from https://genome.jgi.doe.gov/portal/GVMAGs and https://figshare.com/s/14788165283d65466732, and will be available in the Integrated Microbial Genome/Virus (IMG/VR) system ${ }^{74}$ at time of the $v .3 .0$ release. All of the sequence data and metadata from the samples used in this study can further be accessed through the IMG/M system $^{43}$ (https://img.jgi.doe.gov) and NCBISRA using the metagenome identifiers provided in Supplementary Table 1. Sequence alignments, phylogenetic trees and other data underlying this study can be downloaded from https://genome.jgi.doe.gov/portal/GVMAGs.

\section{Code availability}

The NCLDV classifier can be obtained from https://bitbucket.org/ berkeleylab/mtg-gv-exp/.

43. Chen, I. A. et al. IMG/M: integrated genome and metagenome comparative data analysis system. Nucleic Acids Res. 45, D507-D516 (2017).

44. Arndt, W. Modifying HMMER3 to run efficiently on the Cori supercomputer using OpenMP tasking. In Proc. 2018 IEEE International Parallel and Distributed Processing Symposium Workshops (IPDPSW) 239-246 (2018).

45. Li, W. \& Godzik, A. Cd-hit: a fast program for clustering and comparing large sets of protein or nucleotide sequences. Bioinformatics 22, 1658-1659 (2006).

46. Emms, D. M. \& Kelly, S. OrthoFinder: solving fundamental biases in whole genome comparisons dramatically improves orthogroup inference accuracy. Genome Biol. 16, 157 (2015).

47. Buchfink, B., Xie, C. \& Huson, D. H. Fast and sensitive protein alignment using DIAMOND Nat. Methods 12, 59-60 (2015).

48. Katoh, K. \& Standley, D. M. A simple method to control over-alignment in the MAFFT multiple sequence alignment program. Bioinformatics 32, 1933-1942 (2016).

49. Hyatt, D. et al. Prodigal: prokaryotic gene recognition and translation initiation site identification. BMC Bioinformatics 11, 119 (2010).

50. Liaw, A. \& Wiener, M. Classification and regression by randomForest. $R$ News $\mathbf{2}, 18-22$ (2002).

51. R Core Team. R: A Language and Environment for Statistical Computing http://www.Rproject.org/ (R Foundation for Statistical Computing, 2013). (2013).

52. Torchiano, M. effsize: efficient effect size computation. R package version $0.5 .4 \mathrm{https} / / /$ cran.r-project.org/web/packages/effsize/effsize.pdf (2015).

53. Kang, D. D., Froula, J., Egan, R. \& Wang, Z. MetaBAT, an efficient tool for accurately reconstructing single genomes from complex microbial communities. Peer J 3, e1165 (2015).

54. Parks, D. H., Imelfort, M., Skennerton, C. T., Hugenholtz, P. \& Tyson, G. W. CheckM: assessing the quality of microbial genomes recovered from isolates, single cells, and metagenomes. Genome Res. 25, 1043-1055 (2015).

55. Bowers, R. M. et al. Minimum information about a single amplified genome (MISAG) and a metagenome-assembled genome (MIMAG) of bacteria and archaea. Nat. Biotechnol. 35, 725-731 (2017).

56. Kang, D. D. et al. MetaBAT 2: an adaptive binning algorithm for robust and efficient genome reconstruction from metagenome assemblies. PeerJ 7, e7359 (2019).

57. Tully, B. J., Sachdeva, R., Graham, E. D. \& Heidelberg, J. F. 290 metagenome-assembled genomes from the Mediterranean Sea: a resource for marine microbiology. Peer $\mathbf{5}$, e3558 (2017). 


\section{Article}

58. Capella-Gutiérrez, S., Silla-Martínez, J. M. \& Gabaldón, T. trimAl: a tool for automated alignment trimming in large-scale phylogenetic analyses. Bioinformatics 25, 1972-1973 (2009).

59. Nguyen, L.-T., Schmidt, H. A., von Haeseler, A. \& Minh, B. Q. IQ-TREE: a fast and effective stochastic algorithm for estimating maximum-likelihood phylogenies. Mol. Biol. Evol. 32 268-274 (2015).

60. Jain, C., Rodriguez-R, L. M., Phillippy, A. M., Konstantinidis, K. T. \& Aluru, S. High throughput ANI analysis of $90 \mathrm{~K}$ prokaryotic genomes reveals clear species boundaries. Nat. Commun. 9, 5114 (2018)

61. Filée, J. Route of NCLDV evolution: the genomic accordion. Curr. Opin. Virol. 3, 595599 (2013).

62. Borodovsky, M. \& Lomsadze, A. Gene identification in prokaryotic genomes, phages, metagenomes, and EST sequences with GeneMarkS suite. Curr. Protoc. Bioinformatics 35, 4.5.1-4.5.17 (2011).

63. Finn, R. D. et al. The Pfam protein families database: towards a more sustainable future. Nucleic Acids Res. 44, D279-D285 (2016).

64. Nawrocki, E. P. \& Eddy, S. R. Infernal 1.1: 100-fold faster RNA homology searches. Bioinformatics 29, 2933-2935 (2013).

65. Kalvari, I. et al. Rfam 13.0: shifting to a genome-centric resource for non-coding RNA families. Nucleic Acids Res. 46, D335-D342 (2018).

66. Huerta-Cepas, J. et al. Fast genome-wide functional annotation through orthology assignment by eggNOG-Mapper. Mol. Biol. Evol. 34, 2115-2122 (2017).

67. Tabari, E. \& Su, Z. PorthoMCL: parallel orthology prediction using MCL for the realm of massive genome availability. Big Data Analytics 2, 4 (2017).

68. Hoang, D. T., Chernomor, O., von Haeseler, A., Minh, B. Q. \& Vinh, L. S. UFBoot2: improving the ultrafast bootstrap approximation. Mol. Biol. Evol. 35, 518-522 (2018)

69. Kalyaanamoorthy, S., Minh, B. Q., Wong, T. K. F., von Haeseler, A. \& Jermiin, L. S. ModelFinder: fast model selection for accurate phylogenetic estimates. Nat. Methods 14 587-589 (2017).

70. Wu, D. et al. A phylogeny-driven genomic encyclopaedia of Bacteria and Archaea. Nature 462, 1056-1060 (2009).

71. Letunic, I. \& Bork, P. Interactive tree of life (iTOL) v3: an online tool for the display and annotation of phylogenetic and other trees. Nucleic Acids Res. 44, W242-W245 (2016)

72. Price, M. N., Dehal, P. S. \& Arkin, A. P. FastTree 2-approximately maximum-likelihood trees for large alignments. PLoS ONE 5, e9490 (2010).
73. Bastian, M., Heymann, S. \& Jacomy, M. Gephi: an open source software for exploring and manipulating networks. Proc. International AAAI Conference on Weblogs and Social Media (2009)

74. Paez-Espino, D. et al. IMG/VR v.2.0: an integrated data management and analysis system for cultivated and environmental viral genomes. Nucleic Acids Res. 47, D678-D686 (2019).

Acknowledgements This work was conducted by the US Department of Energy Joint Genome Institute, a DOE Office of Science User Facility, under contract no. DE-ACO2-05CH11231 and made use of resources of the National Energy Research Scientific Computing Center, which is also supported by the DOE Office of Science under contract no. DE-ACO2-05CH11231. We thank the DOE JGI user community and Tara Oceans for sampling efforts and for providing the metagenomic data that underlies this study and E. Kirton for running CheckM for non-targeted binning of the IMG/M metagenomes.

Author contributions F.S. and T.W. conceived the study. D.A.W., V.J.D., K.D.M. and K.T.K. provided metagenomic datasets with a large number of GVMAGs. F.S. performed targeted binning of public metagenomes, phylogenomics, analysis of functional genes and HGT analysis. S.R. developed and benchmarked RBS/gene density classifier. D.P.-E. provided initial HMMs for the NCLDV major capsid protein. S.J. performed non-targeted binning of public metagenomes in IMG. F.S. and S.R. performed quality control of GVMAGs. F.S. visualized the data. T.W., N.C.K. and E.A.E.-F. supervised research. F.S. and T.W. prepared the manuscript, with contributions from all authors. All authors read and approved the final manuscript.

Competing interests The authors declare no competing interests.

\section{Additional information}

Supplementary information is available for this paper at https://doi.org/10.1038/s41586-0201957-x.

Correspondence and requests for materials should be addressed to F.S. or T.W.

Peer review information Nature thanks Hisashi Endo, Mart Krupovic and the other, anonymous, reviewer(s) for their contribution to the peer review of this work.

Reprints and permissions information is available at http://www.nature.com/reprints. 


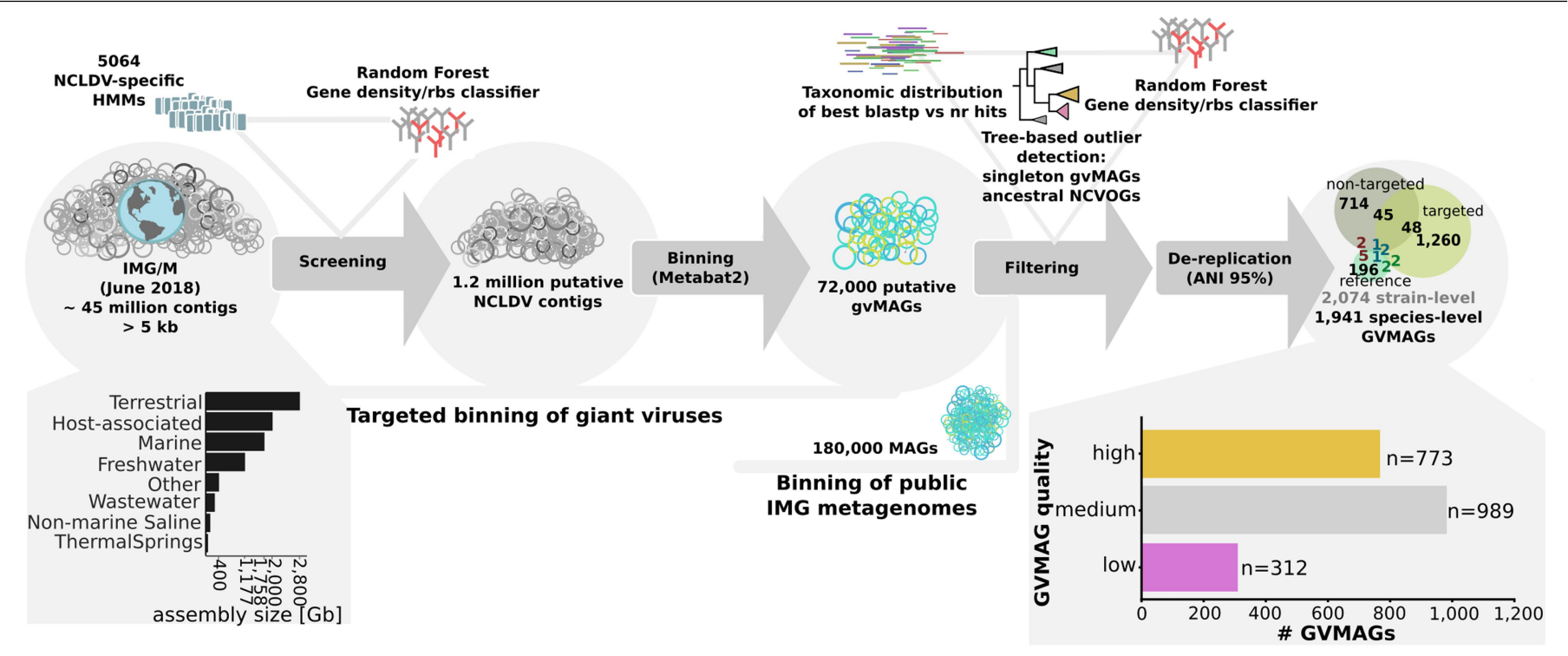

Extended Data Fig. 1 | Discovery pipeline for GVMAGs. Approximately 46 million contigs that were longer than $5 \mathrm{~kb}$ and were available in IMG $/ \mathrm{M}^{15}$ (June 2018) were screened for potential NCLDV contigs using a combination of 5,064 NCLDV-specific HMMs and a random-forest classifier based on gene density and RBS motifs. The resulting set of 1.2 million contigs was then subjected to metagenomic binning using MetaBAT2 ${ }^{53}$, with binning performed separately for each metagenome that contained putative NCLDV contigs. To the resulting approximately 72,000 GVMAGs, we added around 180,000 lowquality MAGs based on MIMAG $^{55}$ that were generated by non-targeted binning

of metagenomes in IMG/M. The resulting set of approximately 252,000 GVMAGs and MAGs were then filtered on the basis of assembly size and using a combination of the consensus of taxonomic affiliation of best blast hits across contigs, the presence or absence and copy numbers of frequently conserved NCLDV genes taking into account neighbouring taxa in the species tree and random-forest classifier based on gene density and RBS motifs. Outlier contigs were removed as described in the Methods and only MAGs that showed a copynumber distribution of frequently conserved NCLDV genes similar to closely related viral genomes were maintained in the final dataset. 
a

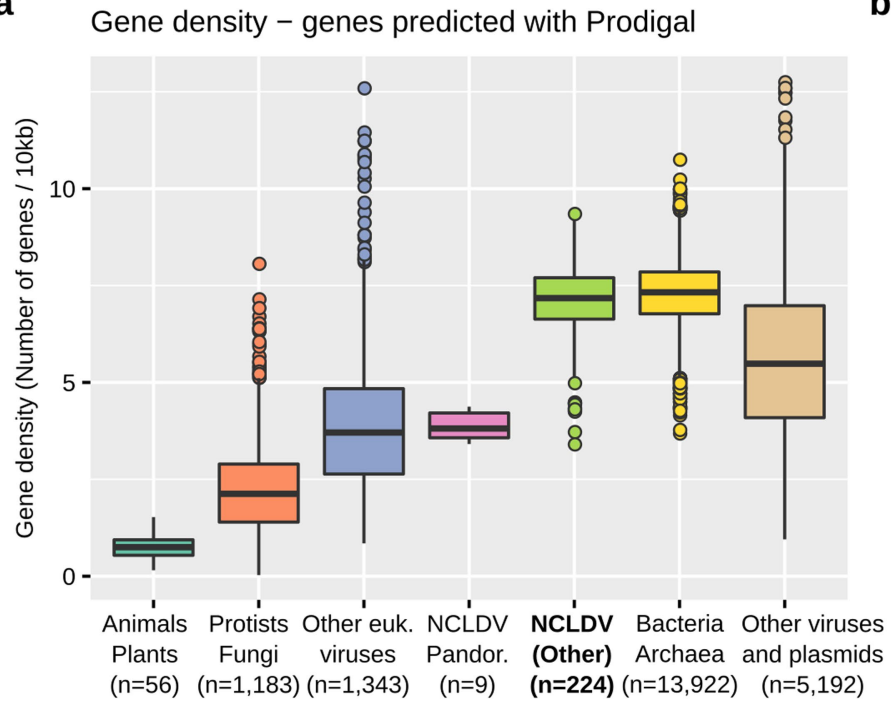

b

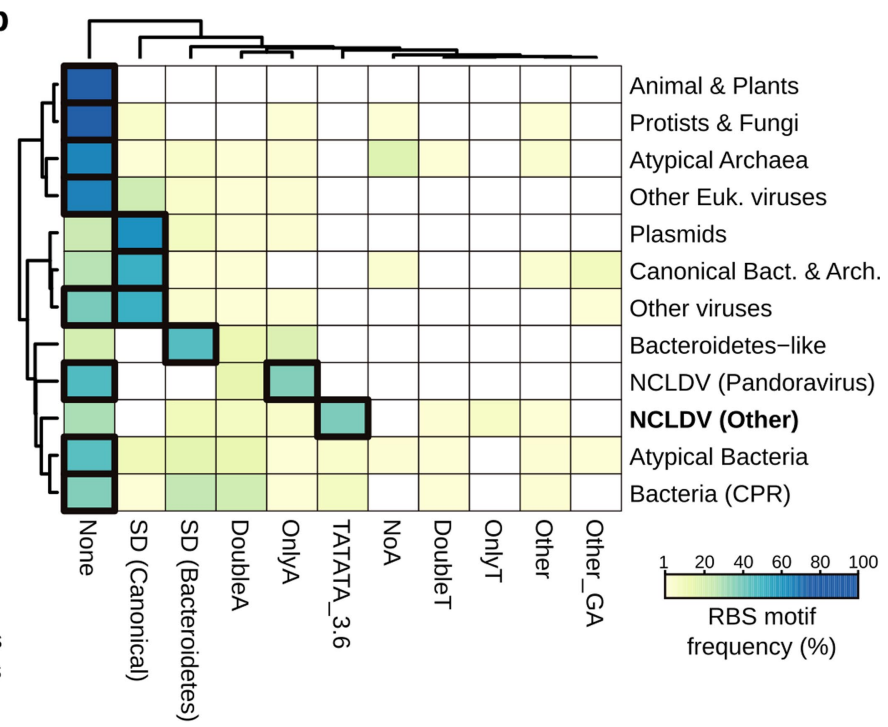

C

NCLDV origin prediction score
0 $(0,0.1]$

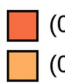

$(0.1,0.2]$
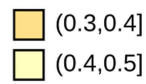

$(0.5,0.6]$ $(0.6,0.7]$
$(0.7,0.8]$

$(0.8,0.9]$

Whole genomes

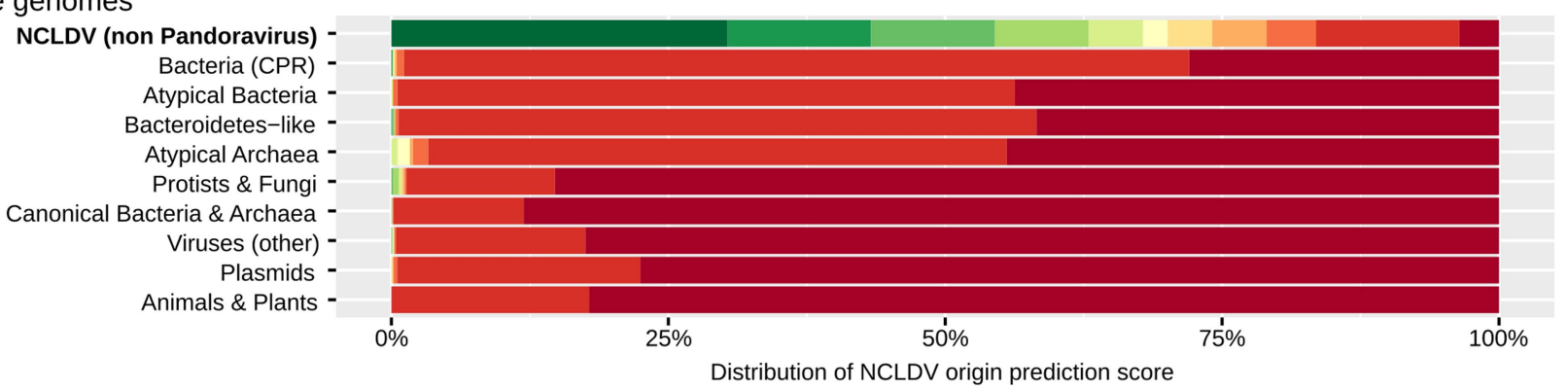

Genome fragments

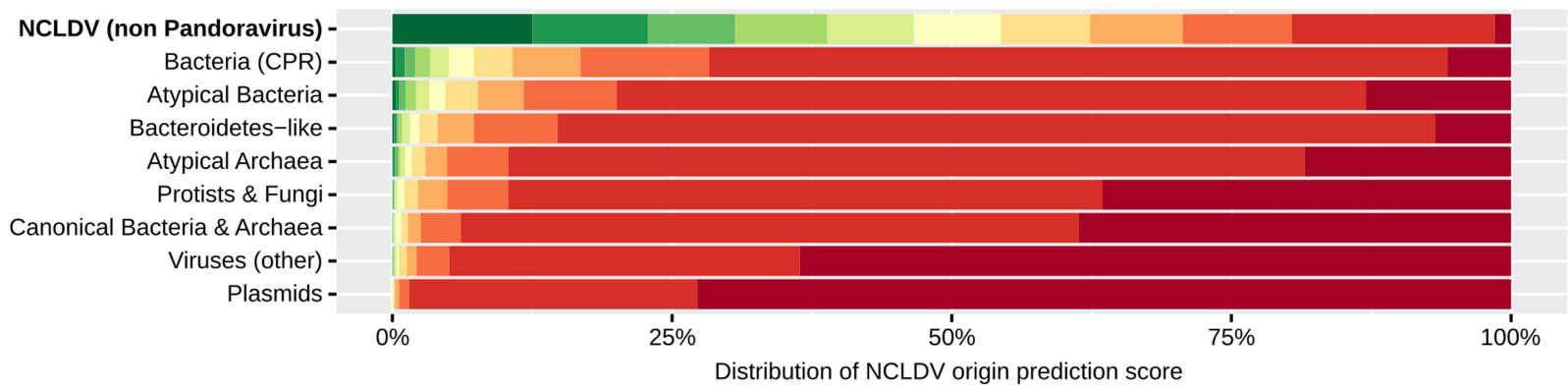

Extended Data Fig. 2 | The RBS classifier. Unique features of NCLDV genomes and efficiency of random-forest classifiers based on these features. a, Gene density ( $y$ axis, average number of genes predicted per $10 \mathrm{~kb}$ of genome) for genomic sequences from different types of organisms or entities ( $x$ axis). Genomes were grouped on the basis of taxonomy (kingdom and domain ranks) as well as patterns of RBS motifs and gene density. 'Other euk. viruses', nonNCLDV eukaryotic viruses; 'NCLDV Pandor.', pandoravirus and similar NCLDVs; 'NCLDV (Other)', non-pandoravirus NCLDVs. Centre lines of box plots represent the median, bounds of the boxes indicate the lower and upper quartiles, whiskers extend to points that lie within $1.5 \times$ the interquartile range of the lower and upper quartiles. Sample sizes (number of genomes) are indicated. b, Frequency of RBS motifs identified across different genomes groups. RBS motif frequencies were based on prodigal gene prediction using the 'full motif scan' option. For clarity, only RBS motif frequencies $>1 \%$ are displayed. RBS motif frequencies $\geq 30 \%$ are highlighted with a bold outline. 'Other Euk. viruses', non-NCLDV eukaryotic viruses; 'NCLDV (pandoravirus)', pandoravirus and similar NCLDVs; 'NCLDV (Other)', non-pandoravirus NCLDVs. c, Predictions of NCLDV origin on the basis of genome features and predicted RBS motifs by random-forest classifiers for complete genomes (top) and short genome fragments (bottom). Predictions for individual genomes were obtained through a tenfold cross-validation. Similar results were obtained when predicting only two classes (NCLDV and non-NCLDV, displayed here) or when predicting classes corresponding to the eight types of genomes. CPR, candidate phyla radiation; SD, Shine-Dalgarno sequence. 
a

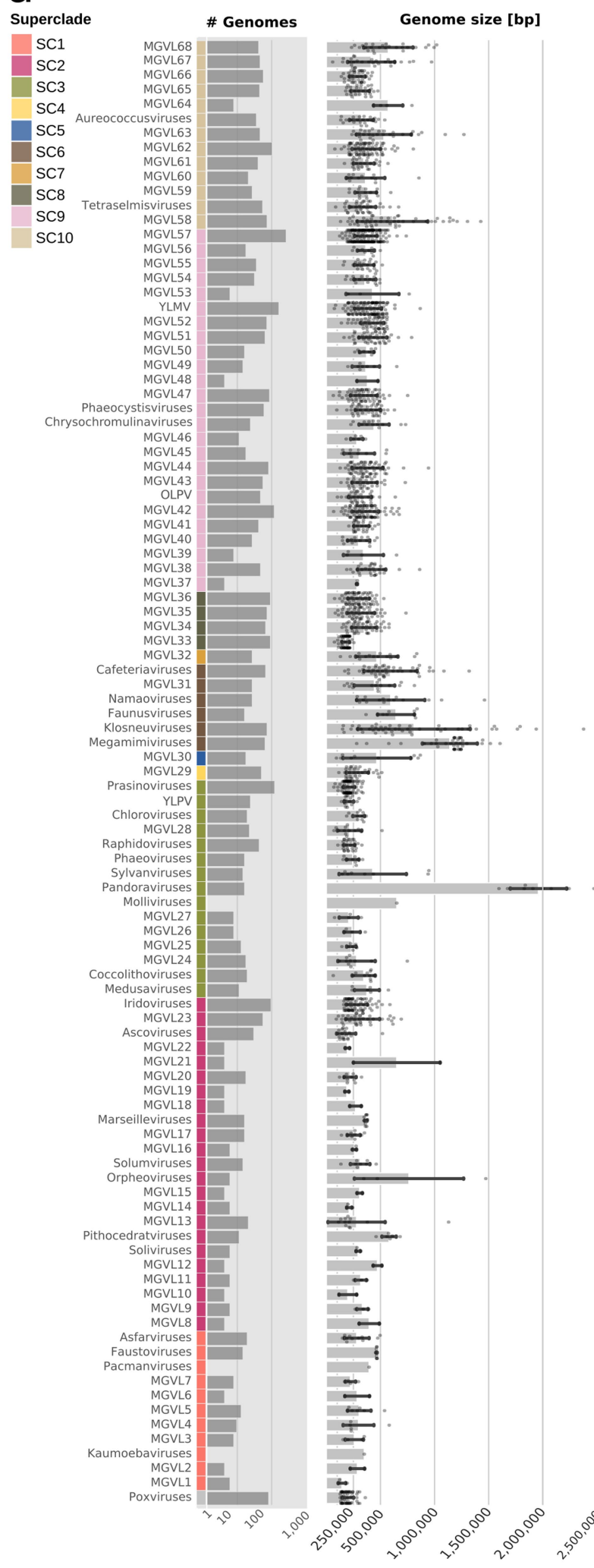

Extended Data Fig. 3 | Features of GVMAGs. a, Mean assembly size, GC content and coding density for each lineage in the NCLDV, coloured by superclade, individual data points are shown. Data are mean \pm s.d. b, Assembly metrics of all GVMAGs compared to previously published NCLDV genomes included in this b

GC [\%] Coding density [\%]

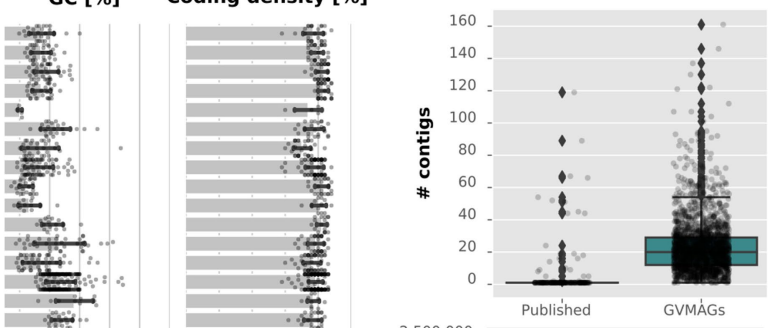

ह ह

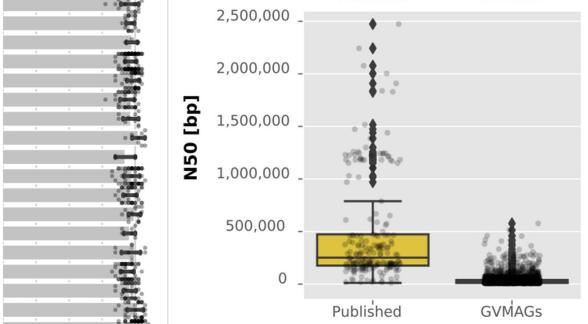

- $=\frac{0}{2}$

की

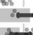

tons:

ti

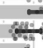

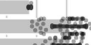

.

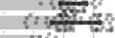

6.

t.

क्ष.

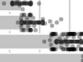

$\tan$

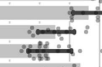

4
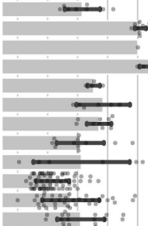

$-$

$-$

$\div$

$\div$

$\div$

...

…ㄴ..1

$\rightarrow$

$\div$

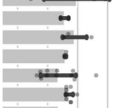

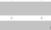
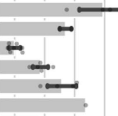

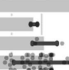
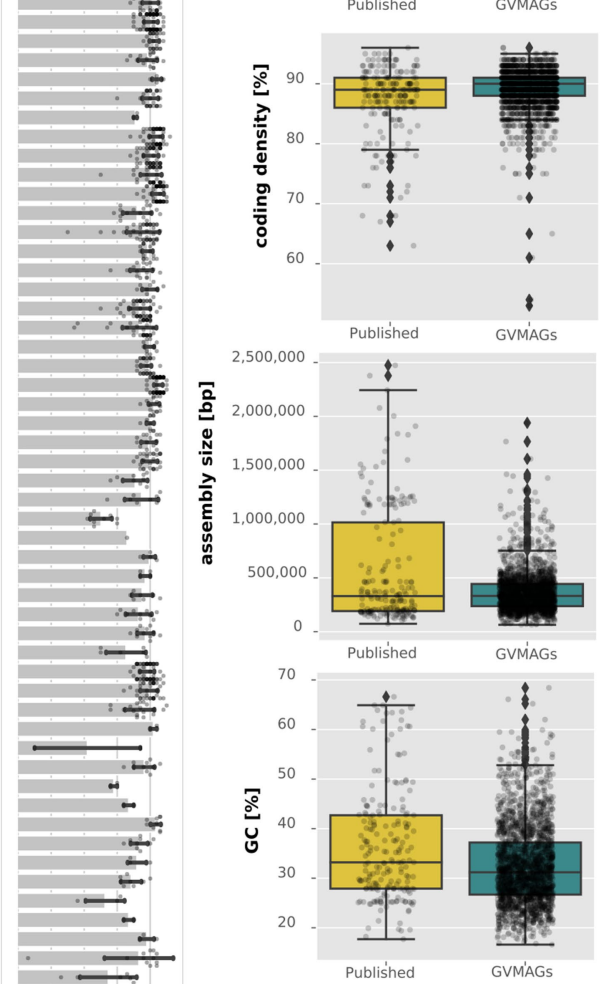

study. Centre lines of box plots represent the median, bounds of boxes indicate the lower and upper quartiles, whiskers extend to points that lie within $1.5 \times$ interquartile range of the lower and upper quartiles. Sample size for the published data is 205 genomes and for GVMAGs is 2,074 genomes. 

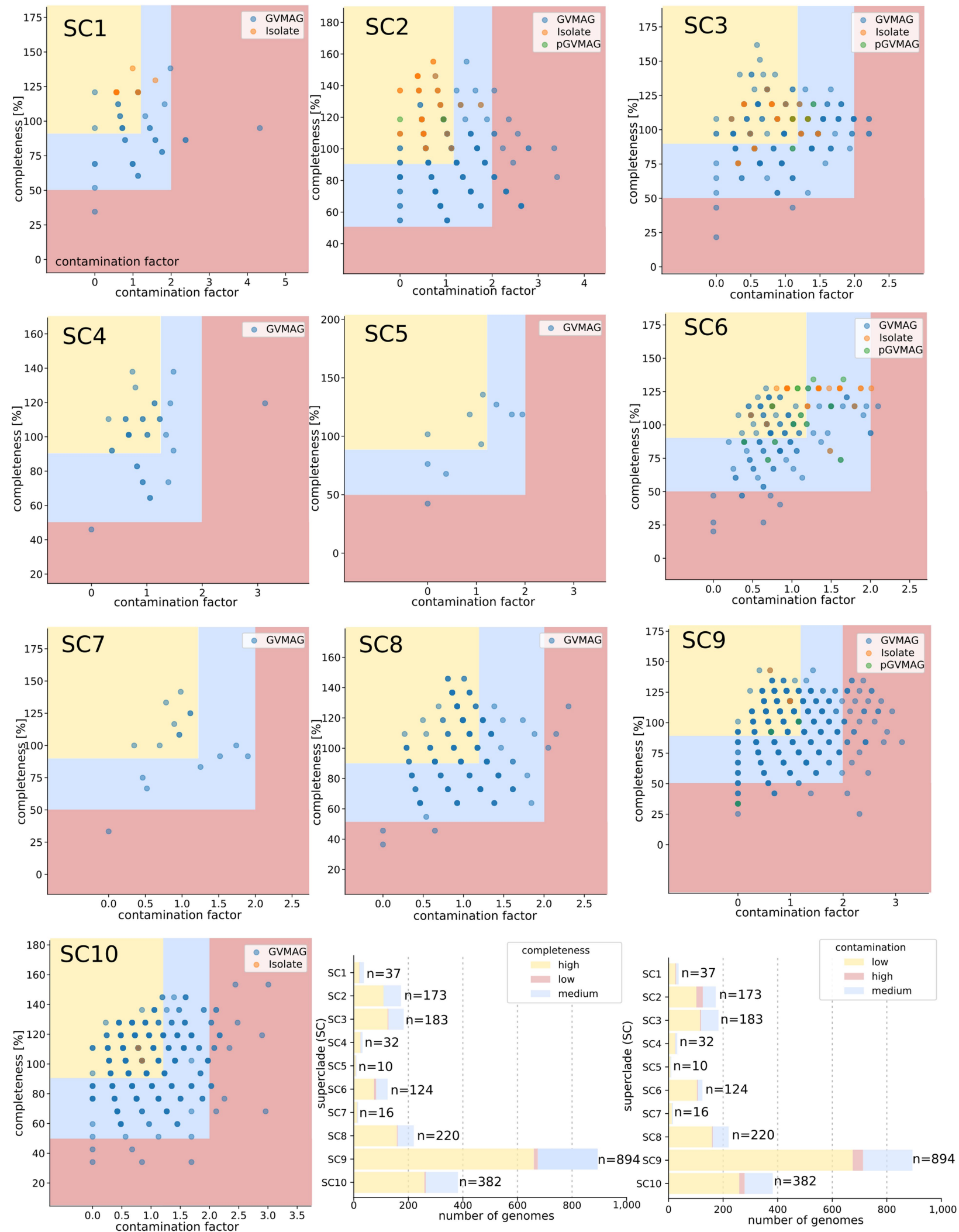

Extended Data Fig. 4 | Estimated completeness and contamination of GVMAGs on the basis of the presence of conserved NCVOGs. Scatter plots show estimated completeness and contamination for GVMAGs in each superclade (SC), previously published GVMAGs (pGVMAGs) and isolate genomes (filled circles with different colours) compared with the average of the respective superclade. Genomes in the red area were classified as low quality, genomes in the blue area were classified as medium quality and genomes in the yellow area were classified as high quality on the basis of the combination of completeness and contamination. Stacked bars (bottom right) summarize, for each NCLDV superclade, the total number of GVMAGs with low, medium and high contamination and completeness. 


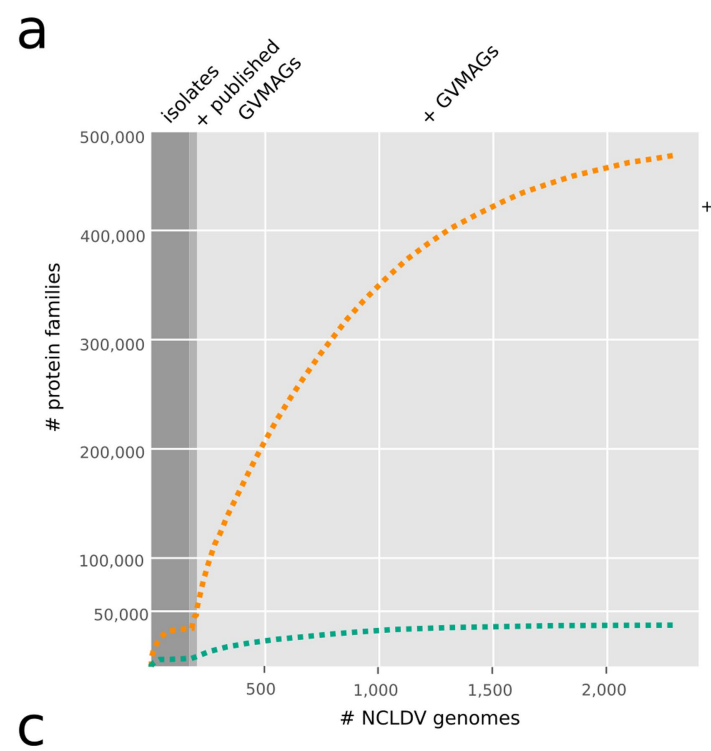

b

C

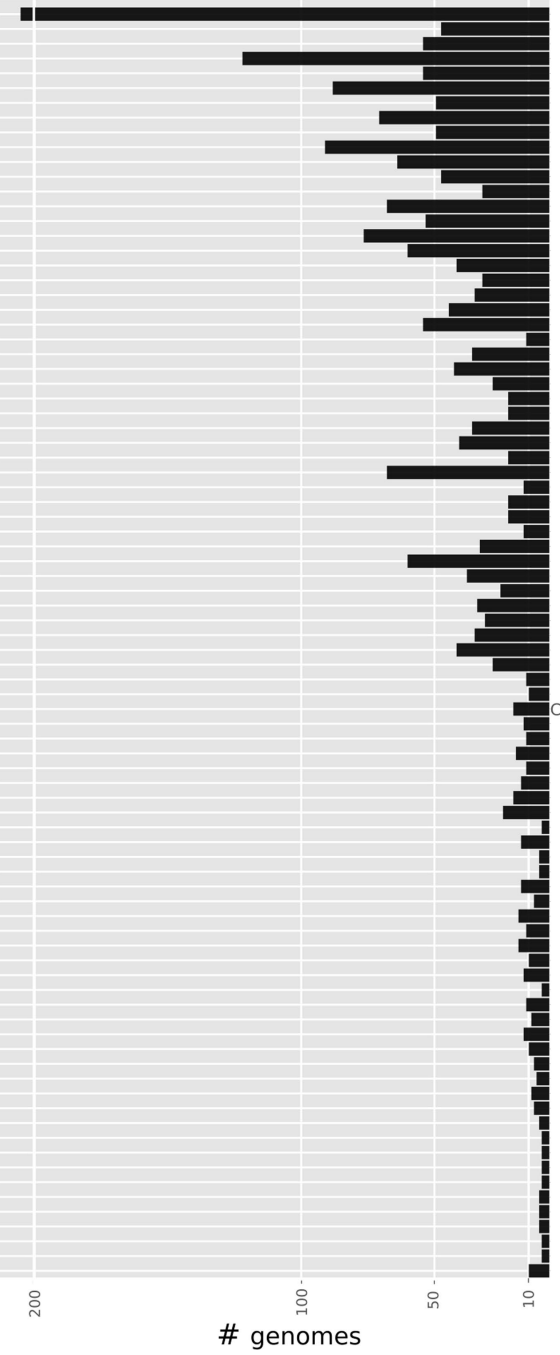

\# genomes

Extended Data Fig. 5 | Shared and unique protein families within NCLDV lineages. a, Collectors curve showing the increase in functional diversity estimated on the basis of the total number of protein families detected in NCLDV isolates, previously published GVMAGs and GVMAGs recovered in this study. The orange curve includes all detected protein families; the blue curve only includes protein families that included by at least two proteins. b. Top, the total number of different Pfam-A domains, total number of proteins with any$$
\text { (1) }
$$

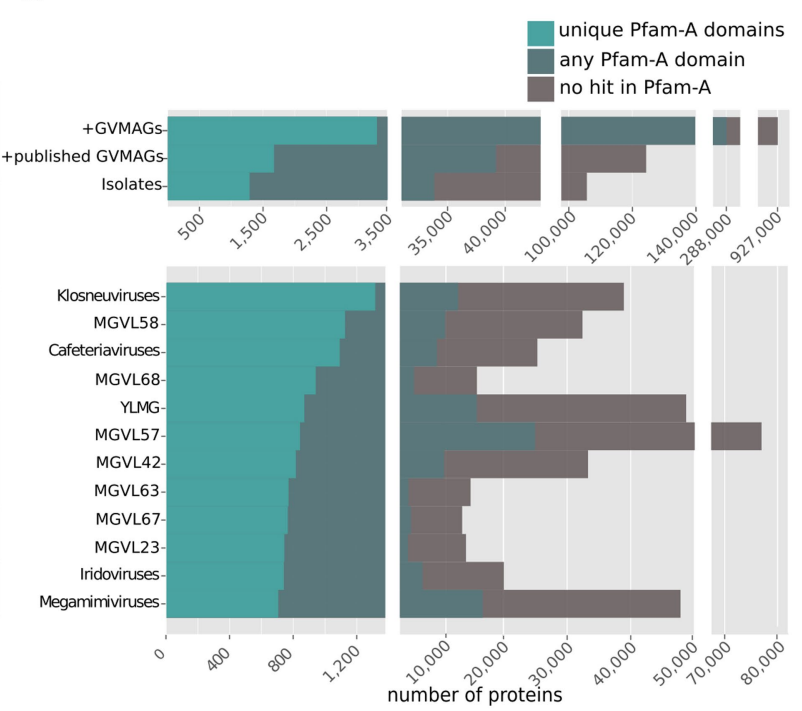

number of protein
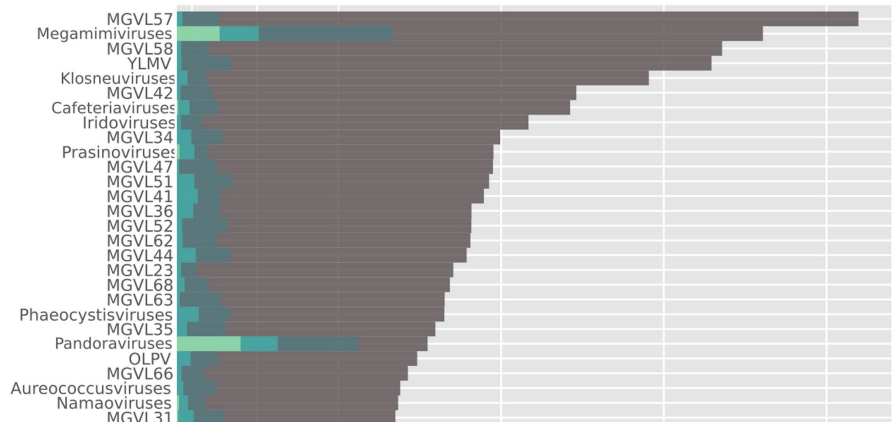

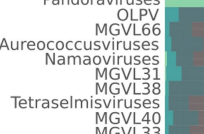
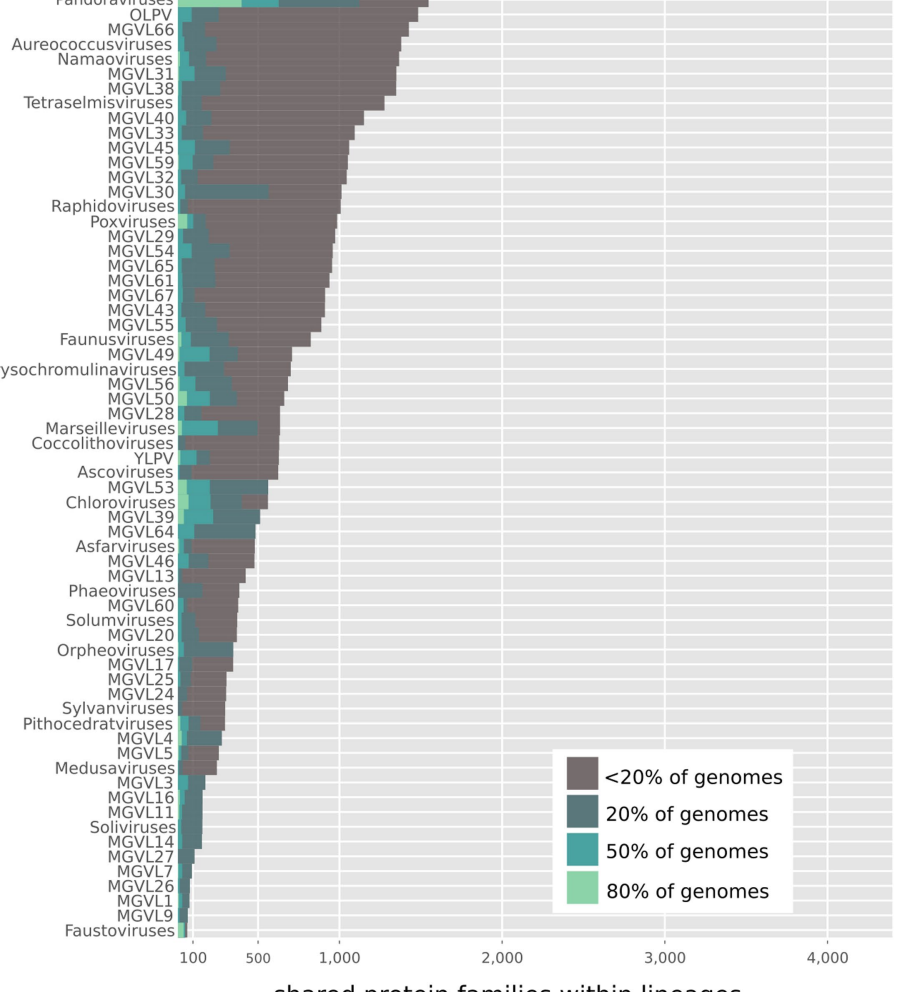

shared protein families within lineages

Pfam-A domain and total number of proteins found in NCLDV isolates, previously published NCLDV genomes from metagenomes and GVMAGs recovered in this study. Bottom, NCLDV lineages with the greatest number of unique Pfam-A domains. c, The total number of genomes per lineage (left) and total number of protein families (at least two members) found in each lineage are indicated together with the proportion of genomes in the respective lineage that share protein families (right). 


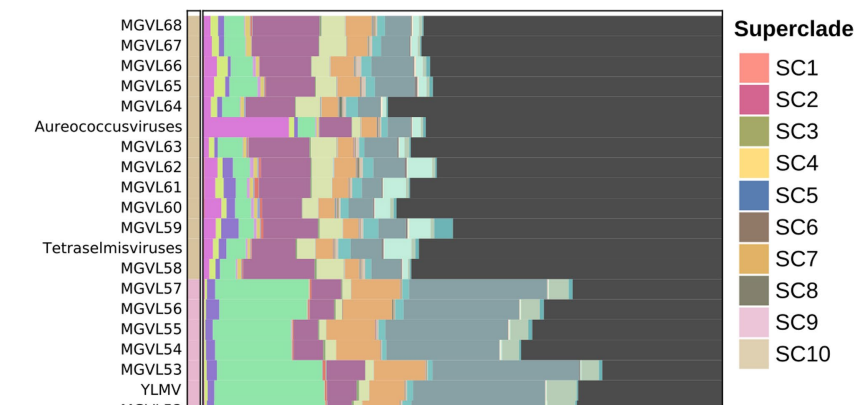

Reference lineages

- Ascoviruses

Asfarviruses

- Aureococcusviruses

Cafeteriaviruses

- Chloroviruses

Chrysochromulinaviruses

Phaeocystisviruses
rysochromulinaviruses
MGVL46

MGVL46
MGVL45

MGVL44

MGVL43

MGVL42

MGVL41

MGVL40

MGVL39

MGVL37

MGVL36

MGVL35

MGVL34

MGVL33

MGVL32

Cafeteriaviruses
MGVL3

MGVL31
aoviruses

Faunusviruses

Klosneuviruses
Megamimiviruses

MGVL30

MGVL29

Prasinoviruse
YLPV

MGVL28

MGVL28
Mhidoviruses

$\begin{array}{r}\text { Raphidoviruses } \\ \text { Phaeoviruses } \\ \hline \text { Syvanius }\end{array}$

Sylvanviruses

Pandoraviruses

Molliviruses

MGVL27

MGVL26

MGVL25
MGVL24

MGVL24
lithoviruses

Medusaviruses

Iridoviruses

MGVL23

Ascoviruses
MGVL22

MGVL22

MGVL20

MGVL19

Marseilleviruses

MGVL17

MGVL16
Solumviruses

Orpheoviruses

MGVL15
MGVL14

MGVL13

Pithocedratviruses

Soliviruses
MGVL12

MGVL12

MGVL10

MGVL10
MGVL9
MGVL8

MGVL8

Asfarviruses
Faustoviruses

Faustoviruses
Pacmanviruses

MGVL7

MGVL6

MGVL4

MGVL3

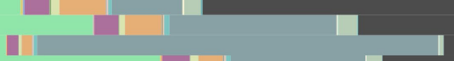

口

1

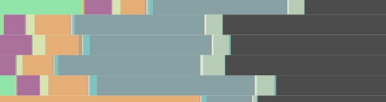

Faunusviruses

Faustoviruses

Iridoviruses

- Kaumoebaviruses

Klosneuviruses

Marseilleviruses

- Medusaviruses

Megamimiviruses

- Molliviruses

Namaoviruses

- OLPV

- Orpheoviruses

Pacmanviruses

- Pandoraviruses

Phaeoviruses

- Pithocedratviruses

Poxviruses

- Prasinoviruses

Phaeocystisviruses

- Raphidoviruses

Soliviruses

Solumviruses

- Sylvanviruses

Tetraselmisviruses

YLMV

YLPV

nohits

MGVL2

MGVL1

Poxviruses

$$
\begin{array}{cccccc}
0 \% & 20 \% & 40 \% & 60 \% & 80 \% & 100 \% \\
& \text { proportion of pangenome with best blastp hits vs known NCDLV lineages } &
\end{array}
$$

Extended Data Fig. 6 | Similarity of proteins encoded in expanded NCLDV lineages and new MGVLs to known NCLDV proteins. For each lineage the proportion of encoded proteins with homology ( $E$-value cut-off of $\left.1 \times 10^{-5}\right)$ to known NCLDV proteins is shown. 
a

Superclade

novel

$\mathrm{SC} 1$

$\mathrm{SC} 2$

$\mathrm{SC} 3$

SC

$\mathrm{SC} 5$

SC

$\mathrm{SC7}$

SC

SC

SC10

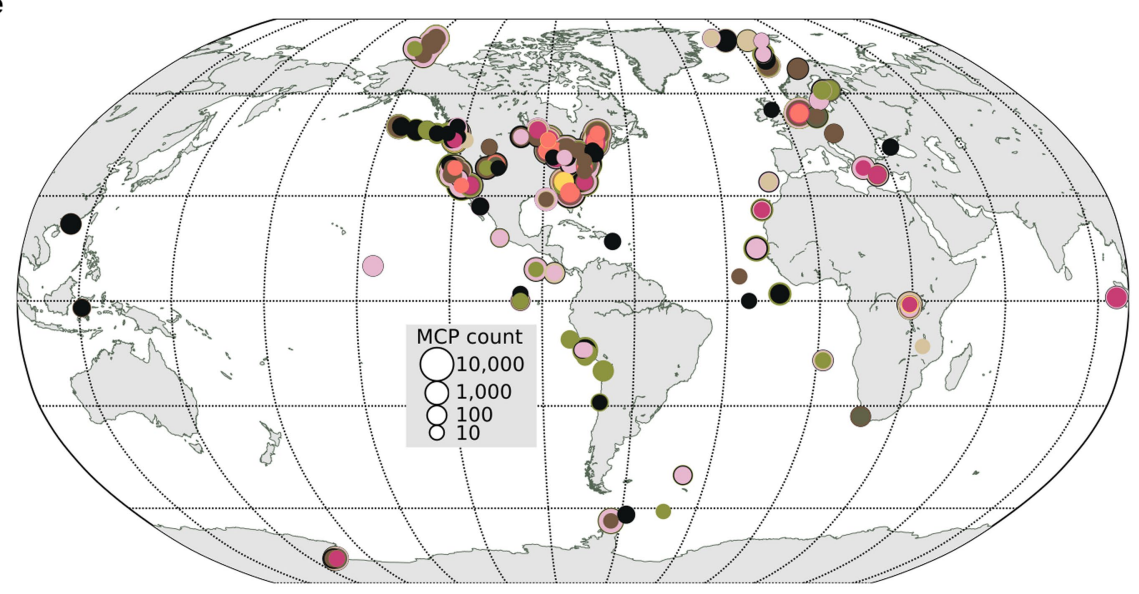

b

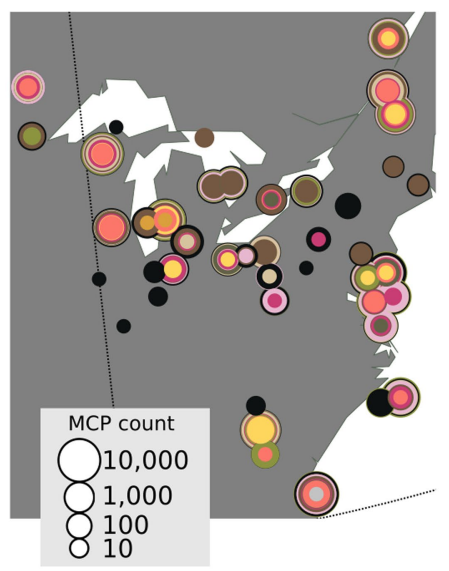

Extended Data Fig. 7 |Distribution of NCLDV MOPs. a, Global distribution of NCLDV MOPs. b, A detailed view of the Midwest and East Coast of the United States and Canada. Filled circles are coloured on the basis of the affiliation with superclade and the circle diameter correlates with the number of MOPs detected at the respective sampling location. Circles at the same coordinates are stacked by size with the largest circles at the bottom. The category 'novel' contains all MCPs that could not be assigned to any of the superclades. 


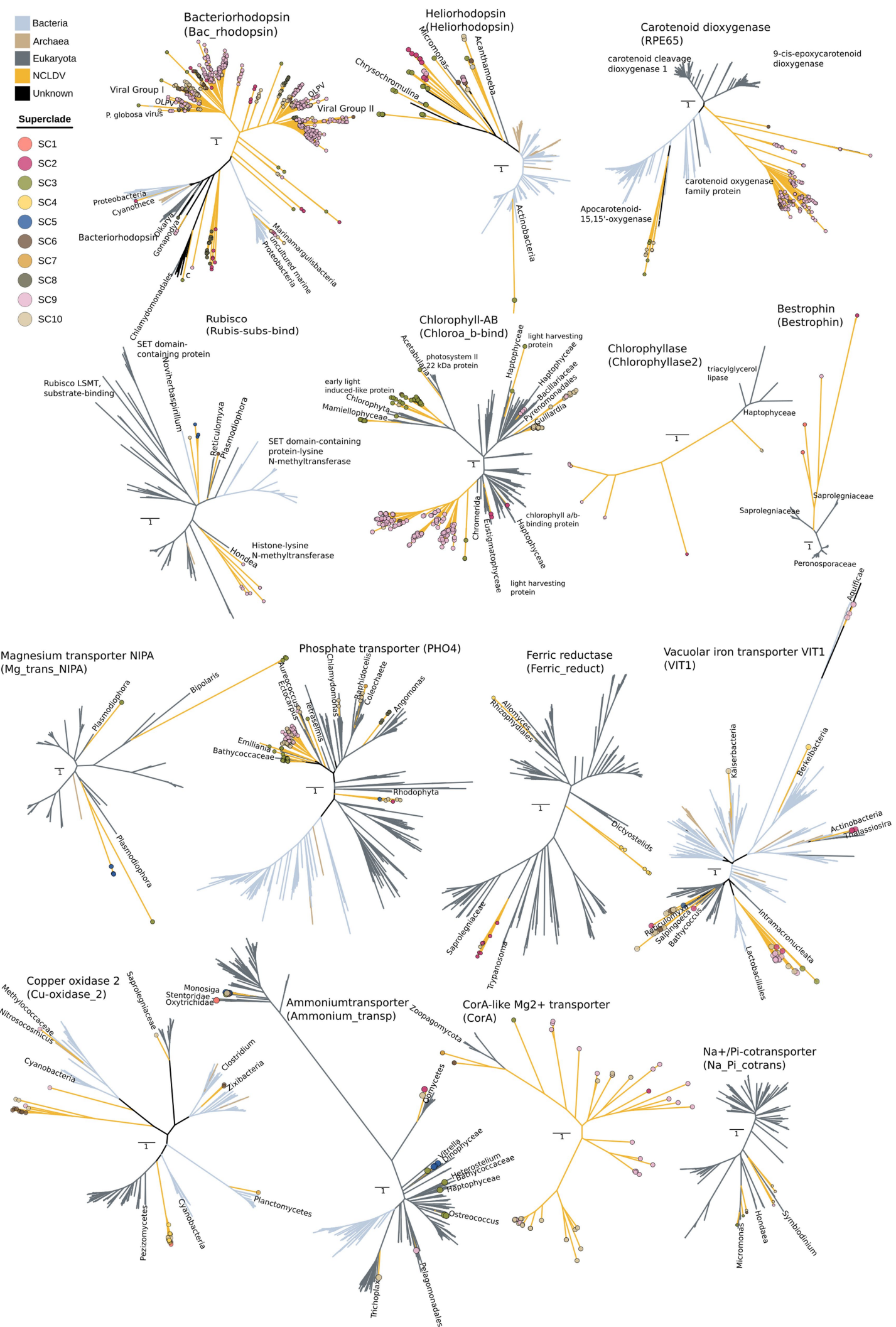

Extended Data Fig. 8 | Maximum-likelihood phylogenetic trees. Maximumlikelihood phylogenetic trees that underlie the analysis in Fig. 2. Trees were inferred using IQ-tree with the following models: $\mathrm{Na}^{+} / \mathrm{P}_{\mathrm{i}}$ cotranporter, LG4M + R7; ammonium transporter, LG4M + R10; bacteriorhodopsin, $\mathrm{LG}+\mathrm{F}+\mathrm{R} 10$; bestrophin, $\mathrm{LG} 4 \mathrm{M}+\mathrm{R} 5$; carotenoid dioxygenase, $\mathrm{LG}+\mathrm{F}+\mathrm{R} 10$;
Chlorophyll ab, LG4M + F+ R10; chlorophyllase, $\mathrm{LG}+\mathrm{I}+\mathrm{G} 4$; CorA-like $\mathrm{Mg}^{2+}$ transporter, LG+F+R3; copper oxidase II, LG4M + R10; heliorhodopsin, LG4M + R9; magnesium transporter NIPA, LG4M + R6; ferric reductase, LG + F+ R9; phosphate transporter, LG4M + R10; Rubisco, LG4M + R6; and vacuolar iron transporter (VIT1), LG4M + R10. 


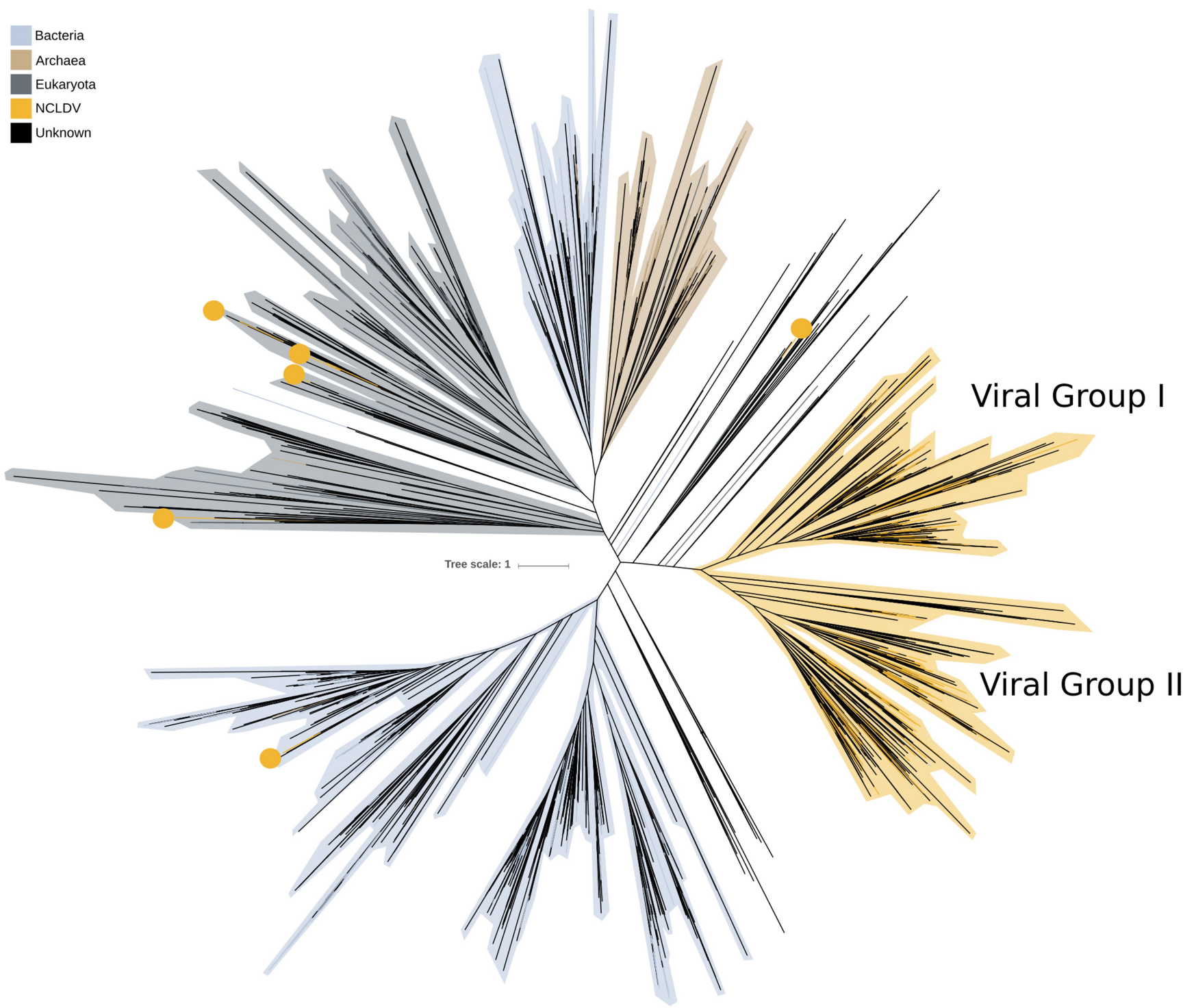

Extended Data Fig. 9 | Diversity of metagenomic rhodopsins. Maximumlikelihood tree (IQ-tree, LG4M + R10 substitution model) of rhodopsins after dereplication through clustering with CD-hit at a 70\% similarity threshold. Clades that predominantly include rhodopsins of archaeal, bacterial, eukaryotic or NCLDV origin are highlighted in the different colours. Yellow filled circles indicate NCLDV rhodopsins that have probably been acquired from cellular organisms through HGT. 


\section{Reporting Summary}

Nature Research wishes to improve the reproducibility of the work that we publish. This form provides structure for consistency and transparency in reporting. For further information on Nature Research policies, seeAuthors \& Referees and theEditorial Policy Checklist .

\section{Statistics}

For all statistical analyses, confirm that the following items are present in the figure legend, table legend, main text, or Methods section.

n/a Confirmed

$\mathbf{x} \square$ The exact sample size $(n)$ for each experimental group/condition, given as a discrete number and unit of measurement

$\mathbf{x} \square$ A statement on whether measurements were taken from distinct samples or whether the same sample was measured repeatedly

$\boldsymbol{x} \square$ The statistical test(s) used AND whether they are one- or two-sided

$x$ Only common tests should be described solely by name; describe more complex techniques in the Methods section.

$\mathbf{x} \square$ A description of all covariates tested

$\mathbf{x} \square$ A description of any assumptions or corrections, such as tests of normality and adjustment for multiple comparisons

$\boldsymbol{x} \square$ A full description of the statistical parameters including central tendency (e.g. means) or other basic estimates (e.g. regression coefficient)

x $\square$ AND variation (e.g. standard deviation) or associated estimates of uncertainty (e.g. confidence intervals)

$\mathbf{x} \square$ For null hypothesis testing, the test statistic (e.g. $F, t, r$ ) with confidence intervals, effect sizes, degrees of freedom and $P$ value noted

$\mathbf{x} \square$ Give $P$ values as exact values whenever suitable.

$\boldsymbol{x} \square$ For Bayesian analysis, information on the choice of priors and Markov chain Monte Carlo settings

$\boldsymbol{x} \square$ For hierarchical and complex designs, identification of the appropriate level for tests and full reporting of outcomes

$\boldsymbol{x} \square$ Estimates of effect sizes (e.g. Cohen's $d$, Pearson's $r$ ), indicating how they were calculated

Our web collection on statistics for biologists contains articles on many of the points above.

\section{Software and code}

Policy information about availability of computer code

\section{Data collection}

IMG/M June2018

NCBI Genbank (June 2018)

NCBI non redundant database May 2019

Pfam-A (v 29.0)

Rfam ( $v$ 13.0)

Data analysis

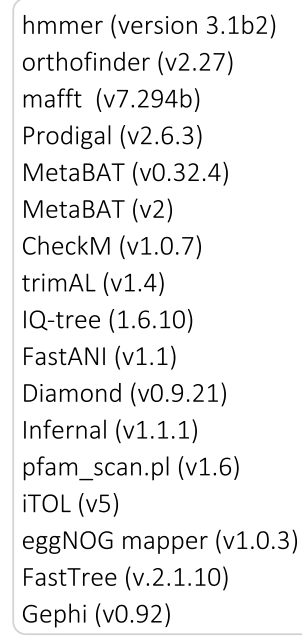


Scikit-learn (v0.20.3)

stats package (v4) in $\mathrm{R}$

PorthoMCL (version of December 2018)

NCLDV classifier is available at https://bitbucket.org/berkeleylab/mtg-gv-exp/

For manuscripts utilizing custom algorithms or software that are central to the research but not yet described in published literature, software must be made available to editors/reviewers. We strongly encourage code deposition in a community repository (e.g. GitHub). See the Nature Research guidelines for submitting code \& software for further information.

Data

Policy information about availability of data

All manuscripts must include a data availability statement. This statement should provide the following information, where applicable:

- Accession codes, unique identifiers, or web links for publicly available datasets

- A list of figures that have associated raw data

- A description of any restrictions on data availability

All GVMAGs of estimated high and medium quality with an N50 of greater than 50kb and estimated 'low' contamination have been deposited at NCBI Genbank under BioProject ID PRJNA588800. Nucleotide and protein sequences of GVMAGs can be directly downloaded from https://genome.jgi.doe.gov/portal/GVMAGs and will become available in IMG/VR74 at time of the v.3.0 release. All the sequence data and metadata from the samples used in this work can further be accessed through the Integrated Microbial Genomes and Microbiomes (IMG/M) systems43 (https://img.jgi.doe.gov) and NCBI SRA using the metagenome identifiers provided in Supplementary Table 1. Sequence alignments, phylogenetic trees and other data underlying this study can be downloaded from https:// genome.jgi.doe.gov/portal/GVMAGs

\section{Field-specific reporting}

Please select the one below that is the best fit for your research. If you are not sure, read the appropriate sections before making your selection.

Life sciences

$\square$ Behavioural \& social sciences

$\boldsymbol{x}$ Ecological, evolutionary \& environmental sciences

For a reference copy of the document with all sections, see nature.com/documents/nr-reporting-summary-flat.pdf

\section{Ecological, evolutionary \& environmental sciences study design}

All studies must disclose on these points even when the disclosure is negative.

Study description

Recovery of nucleocytoplasmic large DNA virus metagenome assembled genomes from all publicly available metagenome data in IMG (https://img.jgi.doe.gov/)

Research sample No samples were taken for this study, subject of this study was all publicly available metagenome data in IMG in June2018 encompassing 8,535 datasets (https://img.jgi.doe.gov/)

Sampling strategy

No samples were taken for this study, all existing publicly available metagenome data in the IMG/M database (https:// img.jgi.doe.gov/) in June 2018 was used in this study.

Data collection The data was collected in June 2018 from the IMG/M database (https://img.jgi.doe.gov/) by Frederik Schulz

Timing and spatial scale Metagenomic data was generated between 2008 and 2018 by the DOE JGI User Community and Tara Oceans

Data exclusions

For unpublished metagenome datasets used in this study, PIs are either included as co-authors, or PIs were asked for permission and if permission was denied the datasets were excluded from the analysis

Reproducibility

This experiment has not been reproduced but can be reproduced with the methods outlined in the manuscript.

Randomization

Randomization in not relevant in this study as all available data has been mined for nucleocytoplasmic large DNA virus metagenome assembled genomes

Blinding

Blinding was not relevant for this study as the same methods have been applied to the entire data set.

Did the study involve field work? $\square$ Yes $\quad \boldsymbol{x}$ No

\section{Reporting for specific materials, systems and methods}

We require information from authors about some types of materials, experimental systems and methods used in many studies. Here, indicate whether each material, system or method listed is relevant to your study. If you are not sure if a list item applies to your research, read the appropriate section before selecting a response. 
Materials \& experimental systems

\begin{tabular}{l|l}
\hline $\mathrm{n} / \mathrm{a}$ & Involved in the study \\
\hline $\boldsymbol{x}$ & $\square$ Antibodies \\
\hline $\boldsymbol{x}$ & $\square$ Eukaryotic cell lines \\
\hline $\boldsymbol{x}$ & $\square$ Palaeontology \\
\hline $\boldsymbol{x}$ & $\square$ Animals and other organisms \\
\hline $\boldsymbol{x}$ & $\square$ Human research participants \\
\hline $\boldsymbol{x}$ & $\square$ Clinical data
\end{tabular}

Methods

$\mathrm{n} / \mathrm{a}$ Involved in the study

$\boldsymbol{x} \square$ ChIP-seq

$\boldsymbol{x} \square$ Flow cytometry

$\boldsymbol{x} \square$ MRI-based neuroimaging 